\title{
Scandium-Catalyzed Allylboration of Aldehydes as a Practical Method for Highly Diastereo- and Enantioselective Construction of Homoallylic Alcohols
}

\author{
Hugo Lachance, Xiaosong Lu, Michel Gravel, and Dennis G. Hall* \\ Department of Chemistry, University of Alberta, Edmonton, AB, T6G 2G2, Canada
}

\section{Supplementary Information}

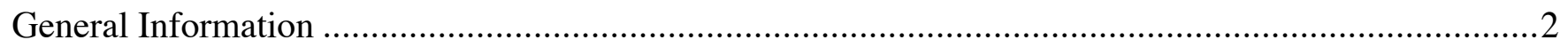

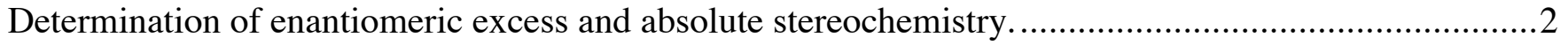

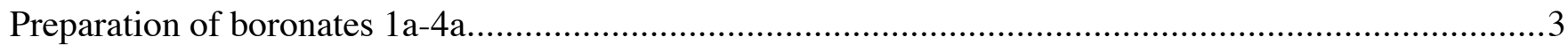

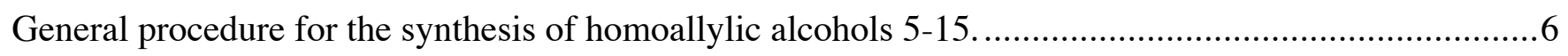

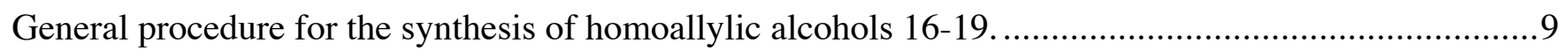

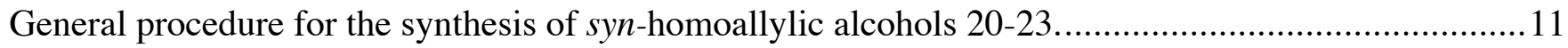

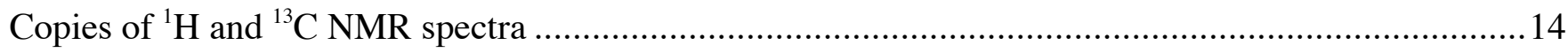

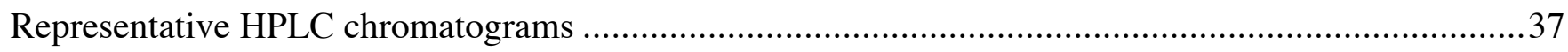

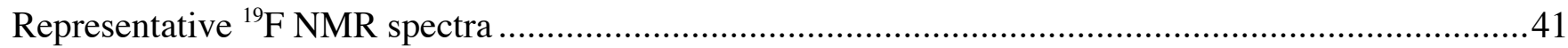




\section{General Information}

Unless otherwise noted, all reactions were performed under an argon atmosphere using flame-dried glassware. Toluene, hexanes and $\mathrm{CH}_{2} \mathrm{Cl}_{2}$ were distilled over $\mathrm{CaH}_{2}$. THF and $\mathrm{Et}_{2} \mathrm{O}$ were distilled over sodium/benzophenone ketyl. All aldehydes were purified by Kugelrohr distillation prior to use. Thin layer chromatography (TLC) was performed on Merck Silica Gel 60 F254 plates and were visualized with UV light and 5\% phosphomolybdic acid/EtOH (PMA). NMR spectra were recorded on Bruker AM 300, Bruker AM 200, Varian INOVA-300, INOVA-400 or INOVA-500 instruments. The residual solvent protons $\left({ }^{1} \mathrm{H}\right)$ or the solvent carbons $\left({ }^{13} \mathrm{C}\right)$ were used as internal standards. Boron NMR spectra are referenced to external $\mathrm{BF}_{3} \cdot \mathrm{OEt}_{2} ;{ }^{19} \mathrm{~F}$ spectra are referenced to external $\mathrm{CFCl}_{3}$ and were run by Glen Bigam of the University of Alberta Spectral Services. ${ }^{1} \mathrm{H}$ NMR data are presented as follows: chemical shift in ppm downfield from tetramethylsilane (multiplicity, coupling constant, integration). The following abbreviations are used in reporting NMR data: s, singlet; br s, broad singlet; d, doublet; t, triplet; q, quartet; qt, quartet of triplets; dd, doublet of doublets; dt, doublet of triplets; $\mathrm{AB}, \mathrm{AB}$ quartet; m, multiplet. High resolution mass spectra were recorded by the University of Alberta Mass Spectrometry Services Laboratory using either electron impact (EI) or electrospray (ES) ionization techniques. Infrared spectra and optical rotations were recorded by University of Alberta Spectral Services and combustion analyses were performed by the University of Alberta Micro-Analytical Lab..

\section{Determination of enantiomeric excess and absolute stereochemistry.}

The enantiomeric excesses for compounds 9, 13, 15 were determined using integration of the ${ }^{19} \mathrm{~F}$ NMR signals of the corresponding Mosher ester derivatives. ${ }^{1}$ The enantiomeric excesses for compounds 5-8, 10-12, 14, 16-18, 20-22 were determined using an HP 1100 HPLC system. The enantiomeric excesses for compounds 19 and 23 were determined on the corresponding phenylisocyanate adduct using an HP 1100 HPLC system. Columns Chiralcel AD-RH, Chiralcel OD-RH, Chiralcel OD, and Chiralcel AD were purchased from Chiral Technologies Inc.. Racemic homoallylic alcohols were prepared in the same manner using the pinacol boronate derivatives. The absolute stereochemistry for compounds $16,17,18,20$, and 22 was determined by comparison of the sign of optical rotation with reported literature values. The absolute stereochemistry for all other homoallylic alcohols was assigned by analogy.

${ }^{1}$ Dale, J.A.; Dull, D.L.; Mosher, H.S. J. Org. Chem. 1969, 34, 2543. 


\section{Preparation of boronates 1a-4a}

$(1 R, 2 S, 3 R, 4 S)-2,3-0-\left[\right.$ Allylboryl]-2-phenyl-1,7,7-trimethylbornanediol (1a).. ${ }^{2}$

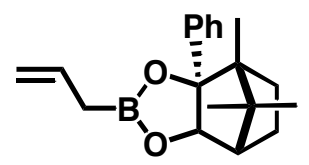

To a solution of triethylborate $(1.30 \mathrm{~mL}, 5.71 \mathrm{mmol})$ in $\mathrm{Et}_{2} \mathrm{O}(12 \mathrm{~mL})$ at $-78^{\circ} \mathrm{C}$ was added a solution of allylmagnesium bromide $\left(1.02 \mathrm{M}\right.$ in $\left.\mathrm{Et}_{2} \mathrm{O}, 5.00 \mathrm{ml}, 5.14 \mathrm{mmol}\right)$ dropwise and the resulting mixture was stirred at $-78^{\circ} \mathrm{C}$ for 4 hours. The resulting suspension was poured onto an ice-cold mixture of $1 \mathrm{~N}$ $\mathrm{HCl}(50 \mathrm{~mL}), \mathrm{Et}_{2} \mathrm{O}(50 \mathrm{~mL})$, and the diol 24. The resulting mixture was stirred at ambient temperature for 30 minutes, then extracted with $\mathrm{Et}_{2} \mathrm{O}(3 \square 25 \mathrm{~mL})$. The combined organic layers were washed with brine, dried over $\mathrm{MgSO}_{4}$, filtered, and concentrated under reduced pressure. Flash chromatography (10\% EtOAc/hexanes) yielded a colourless oil (1.104 g, 93\%), which gave satisfactory analytical data. ${ }^{3}$ TLC (15\% EtOAc/hexanes, PMA): 0.60;

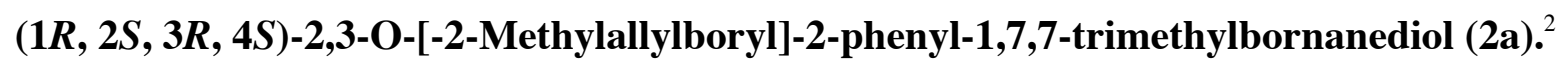

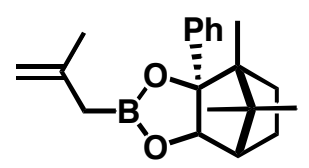

To a solution of triethylborate $(1.50 \mathrm{~mL}, 6.60 \mathrm{mmol})$ in $\mathrm{Et}_{2} \mathrm{O}(12 \mathrm{ml})$ was added diol $24(1.48 \mathrm{~g}$, $6.00 \mathrm{mmol})$. The mixture was stirred at ambient temperature for 30 minutes, then concentrated on the rotary evaporator and co-evaporated with $\mathrm{CH}_{2} \mathrm{Cl}_{2}(3 \square)$. The remaining colourless oil was dissolved in $\mathrm{Et}_{2} \mathrm{O}(12 \mathrm{ml})$ and cooled to $-78^{\circ} \mathrm{C}$. To this solution was added a solution of 2-methylallylmagnesium chloride $(0.5 \mathrm{M}$ in THF, $12.6 \mathrm{ml}, 6.3 \mathrm{mmol})$. The resulting suspension was stirred at $-78^{\circ} \mathrm{C}$ for 4 hours, then poured on an ice-cold mixture of $1 \mathrm{~N} \mathrm{HCl}(50 \mathrm{~mL})$ and $\mathrm{Et}_{2} \mathrm{O}(50 \mathrm{ml})$. The resulting mixture was stirred at ambient temperature for 30 minutes, then extracted with $\mathrm{Et}_{2} \mathrm{O}(3 \square 25 \mathrm{ml})$. The combined organic layers were combined, washed with brine, dried over $\mathrm{MgSO}_{4}$, filtered, and concentrated under reduced pressure. Flash chromatography (10\% EtOAc/hexanes, $\mathrm{SiO}_{2}$ pre-treated with $\left.5 \% \mathrm{Et}_{3} \mathrm{~N} / \mathrm{hexanes}\right)$ yielded a colourless oil $(1.31 \mathrm{~g}, 70 \%)$.

TLC (15\% EtOAc/hexanes, PMA): 0.60;

${ }^{1} \mathrm{H}$ NMR (300 MHz, $\mathrm{CDCl}_{3}$ ): 2 7.44-7.41 (m, 2H), 7.37-7.25 (m, 3H), $4.73(\mathrm{~s}, 1 \mathrm{H}), 4.62$ (dd, J= 1.9 Hz, $1.4 \mathrm{~Hz}, 1 \mathrm{H}), 4.57(\mathrm{~d}, J=0.8 \mathrm{~Hz}, 1 \mathrm{H}), 1.88-1.76(\mathrm{~m}, 1 \mathrm{H}), 1.75(\mathrm{dd}, J=1.4 \mathrm{~Hz}, 0.8 \mathrm{~Hz}, 3 \mathrm{H}), 1.71(\mathrm{~s}, 2 \mathrm{H})$, $1.24(\mathrm{~s}, 3 \mathrm{H}), 1.27-1.14(\mathrm{~m}, 2 \mathrm{H}), 1.08-0.98(\mathrm{~m}, 1 \mathrm{H}), 0.96(\mathrm{~s}, 3 \mathrm{H}), 0.94(\mathrm{~s}, 3 \mathrm{H})$;

\footnotetext{
2 This method was inspired by that of Roush and co-workers: Roush, W.R.; Ando, K.; Powers, D.B.; Palkowitz, A.D.; Halterman, R.L. J. Am. Chem. Soc. 1990, 112, 6339-6348.

3 Herold, T.; Schrott, U.; Hoffmann, R.W. Chem. Ber. 1981, 114, 359-374.
} 
${ }^{15} \mathrm{C}$ NMR $\left(125 \mathrm{MHz}, \mathrm{CDCl}_{3}\right): \partial$ 142.8, 141.8, 127.5, 127.3, 126.8, 124.8, 110.0, 100.5, 95.8, 88.7, 52.0, 50.2, 48.9, 29.6, 24.8, 24.4, 23.6, 20.9, 9.4;

${ }^{11} \mathrm{~B}\left(128 \mathrm{MHz}, \mathrm{CDCl}_{3}\right): \square 33.6$

IR $\left(\mathrm{CH}_{2} \mathrm{Cl}_{2}\right.$ cast, $\left.\mathrm{cm}^{-1}\right): 3072,2957,1646,1445,1338,1034$;

HRMS (EI, $m / z)$ Calcd for $\mathrm{C}_{20} \mathrm{H}_{27} \mathrm{O}_{2} \mathrm{~B}: 310.21042$. Found: 310.21114;

Anal. Calcd for $\mathrm{C}_{20} \mathrm{H}_{27} \mathrm{O}_{2} \mathrm{~B}$ : C, 77.43; H, 8.77. Found: C, 77.40; H, 8.85;

$[\square]_{\mathrm{D}}^{25}+24.3^{\circ}\left(c=2.16, \mathrm{CHCl}_{3}\right)$.

$(1 R, 2 S, 3 R, 4 S)-2,3-0-[(E)-2-b u t e n y l b o r y l]-2-p h e n y l-1,7,7-t r i m e t h y l b o r n a n e d i o l ~(3 a){ }^{2}$

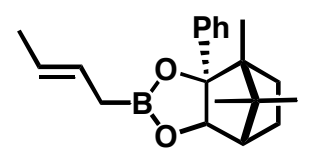

A 200-mL three-neck round-bottom flask equipped with a magnetic stir bar and a thermometer was charged with $110 \mathrm{~mL}$ of THF and $\mathrm{KOtBu}(4.65 \mathrm{~g}, 41.5 \mathrm{mmol})$. This mixture was flushed with Ar and cooled to $-78^{\circ} \mathrm{C}$, and then trans-2-butene $(2.46 \mathrm{~g}, 43.9 \mathrm{mmol})$, condensed into a rubber-stoppered $10-\mathrm{ml}$ round-bottom flask kept at $-78^{\circ} \mathrm{C}$, was added via cannula. $n$-BuLi (1.43 $\mathrm{M}$ in hexane, $\left.29 \mathrm{~mL}, 41.5 \mathrm{mmol}\right)$ was then added dropwise over 1 hour with syringe pump, so that the internal temperature did not rise at all. After completion of the addition, the reaction mixture was allowed to warm until the internal temperature reached $-52^{\circ} \mathrm{C}$. The solution was maintained at $-52^{\circ} \mathrm{C}$ for 15 minutes, then cooled back to $78^{\circ} \mathrm{C}$. Triisopropylborate $(10.53 \mathrm{~mL}, 45.6 \mathrm{mmol})$ was then added dropwise over 30 minutes through a syringe pump. The reaction mixture was maintained at $-78^{\circ} \mathrm{C}$ for 2 hours, then sealed with Ar and stored in freezer for a few weeks without any noticeable change of its quality.

To make reaction boronate $\mathbf{3 a}, 10 \mathrm{~mL}$ of the stored solution was reacted with $15 \mathrm{~mL}$ of $1 \mathrm{~N} \mathrm{HCl}$, and extracted by ether three times. The combined organic layers were mixed with $220 \mathrm{mg}$ of diol 24 and was stirred at ambient temperature for 45 minutes, then concentrated under reduced pressure. Flash chromatography $\left(5 \%\right.$ EtOAc/hexanes, $\mathrm{SiO}_{2}$ pre-treated with $5 \% \mathrm{Et}_{3} \mathrm{~N} /$ hexanes) yielded a colourless oil $277 \mathrm{mg}$ (99\% yield).

${ }^{1} \mathrm{H}$ NMR (500 MHz, $\left.\mathrm{CDCl}_{3}\right):$ 2 7.42-7.41 (m, 2H), 7.34-7.24 (m, 3H), 5.43-5.30 (m, 2H), $4.71(\mathrm{~s}, 1 \mathrm{H})$, $2.13(\mathrm{~d}, J=5.5 \mathrm{~Hz}, 1 \mathrm{H}), 1.84-1.78(\mathrm{~m}, 1 \mathrm{H}), 1.61-1.52(\mathrm{~m}, 5 \mathrm{H}), 1.20-1.13(\mathrm{~m}, 5 \mathrm{H}), 1.04-0.94(\mathrm{~m}, 1 \mathrm{H})$, $0.93(\mathrm{~s}, 3 \mathrm{H}), 0.92(\mathrm{~s}, 3 \mathrm{H})$;

${ }^{13} \mathrm{C}$ NMR (125 MHz, $\left.\mathrm{CDCl}_{3}\right): \partial 141.8,127.4,127.2,126.7,125.7,125.2,95.6,88.5,52.0,50.2,48.8$, 29.6, 24.8, 23.6, 20.7, 18.0, 9.3;

${ }^{11} \mathrm{~B}$ NMR (64 MHz, $\left.\mathrm{CDCl}_{3}\right):$ ə 34.2;

IR $\left(\mathrm{CH}_{2} \mathrm{Cl}_{2}\right.$ cast, $\left.\mathrm{cm}^{-1}\right): 3001,2957,1348,1319,1265,1011$;

HRMS (EI, $m / z$ ) Calcd for $\mathrm{C}_{20} \mathrm{H}_{27} \mathrm{O}_{2} \mathrm{~B}: 310.21042$. Found: 310.20994; 
Anal. Calcd for $\mathrm{C}_{20} \mathrm{H}_{27} \mathrm{O}_{2} \mathrm{~B}$ : C, 77.43; H, 8.77. Found: C, 77.24; H, 8.86;

$[\square]_{D}^{25}=+10.5^{\circ}\left(c=1.84, \mathrm{CHCl}_{3}\right)$.

$(1 R, 2 S, 3 R, 4 S)-2,3-0-[(Z)-2-b u t e n y l b o r y l]-2-p h e n y l-1,7,7-t$ rimethylbornanediol $(4 a){ }^{2}$

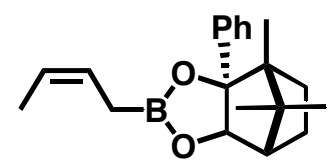

A 300-mL three-neck round-bottom flask equipped with a magnetic stir bar and a thermometer was charged with $110 \mathrm{~mL}$ of THF and KOtBu (2.86 g, $25.5 \mathrm{mmol})$. This mixture was flushed with Ar and cooled to $-78^{\circ} \mathrm{C}$, and then cis-2-butene $(1.50 \mathrm{~g}, 26.8 \mathrm{mmol})$, condensed into a rubber-stoppered $10-\mathrm{ml}$ round-bottom flask kept at $-78^{\circ} \mathrm{C}$, was added via cannula. $n$-BuLi (1.43 $\mathrm{M}$ in hexane, $17.9 \mathrm{~mL}, 25.5$ mmol) was then added dropwise over 1 hour, such that the internal temperature did not rise above $-70^{\circ} \mathrm{C}$. After completion of the addition, the cooling bath was removed and the reaction mixture was allowed to warm up until the internal temperature reached $-25^{\circ} \mathrm{C}$. The solution was maintained at $-25^{\circ} \mathrm{C}$ for 30 minutes, then cooled back to $-78^{\circ} \mathrm{C}$. Triisopropylborate $(6.48 \mathrm{~mL}, 28.1 \mathrm{mmol})$ was then added dropwise over 30 minutes. The reaction mixture was maintained at $-78^{\circ} \mathrm{C}$ for 2 hours, then rapidly poured into a $500-\mathrm{mL}$ separatory funnel containing $200 \mathrm{~mL}$ of $1 \mathrm{~N} \mathrm{HCl}$. The layers were separated, then the aqueous layer was extracted with $\mathrm{Et}_{2} \mathrm{O}(2 \square 100 \mathrm{~mL})$. The combined organic layers were dried over $\mathrm{MgSO}_{4}$ and filtered before being concentrated down on the rotary evaporator to a volume of $63 \mathrm{~mL}$. The resulting solution was approximately $0.4 \mathrm{M}$ in (Z)-2-butenylboronic acid, and could be kept at $4^{\circ} \mathrm{C}$ for a few weeks without any noticeable change in its concentration or its reactivity with diols.

To this solution $(3.00 \mathrm{~mL}, 1.20 \mathrm{mmol})$ was added diol $24(246 \mathrm{mg}, 1.00 \mathrm{mmol})$ and the resulting mixture was stirred at ambient temperature for 30 minutes, then concentrated under reduced pressure. Flash chromatography $\left(10 \% \mathrm{EtOAc} /\right.$ hexanes, $\mathrm{SiO}_{2}$ pre-treated with $5 \% \mathrm{Et}_{3} \mathrm{~N} /$ hexanes $)$ yielded a colourless oil (307 mg, 99\%).

TLC (15\% EtOAc/hexanes, PMA): 0.58;

${ }^{1} \mathrm{H}$ NMR (300 MHz, $\left.\mathrm{CDCl}_{3}\right)$ : 2 7.44-7.38 (m, 2H), 7.35-7.22 (m, 3H), 5.52-5.34 (m, 2H) $4.70(\mathrm{~s}, 1 \mathrm{H})$, $2.13(\mathrm{~d}, J=5.2 \mathrm{~Hz}, 1 \mathrm{H}), 1.87-1.74(\mathrm{~m}, 1 \mathrm{H}), 1.66-1.61(\mathrm{~m}, 2 \mathrm{H}), 1.54-1.50(\mathrm{~m}, 3 \mathrm{H}), 1.21(\mathrm{~s}, 3 \mathrm{H}), 1.21-$ $1.10(\mathrm{~m}, 2 \mathrm{H}), 1.07-0.96(\mathrm{~m}, 1 \mathrm{H}), 0.94(\mathrm{~s}, 3 \mathrm{H}), 0.91(\mathrm{~s}, 3 \mathrm{H})$;

${ }^{13} \mathrm{C}$ NMR (125 MHz, $\left.\mathrm{CDCl}_{3}\right): \partial 141.8,127.4,127.2,126.7 .124 .9,123.5,95.7,88.6,52.0,50.2,48.9$, 29.6, 24.7, 23.6, 20.7, 12.5, 9.3;

${ }^{11} \mathrm{~B}$ NMR $\left(128 \mathrm{MHz} \mathrm{CDCl}_{3}\right):$ ə 34.1

IR $\left(\mathrm{CH}_{2} \mathrm{Cl}_{2}\right.$ cast, $\left.\mathrm{cm}^{-1}\right): 3020,2957,1446,1340,1035$;

HRMS (EI, $m / z$ ) Calcd for $\mathrm{C}_{20} \mathrm{H}_{27} \mathrm{O}_{2} \mathrm{~B}: 310.21042$. Found: 310.21036;

Anal. Calcd for $\mathrm{C}_{20} \mathrm{H}_{27} \mathrm{O}_{2} \mathrm{~B}$ : C, 77.43; H, 8.77. Found: C, 77.41; H, 8.77; 
$[\square]^{2 د}+12.8^{\circ}\left(c=1.73, \mathrm{CHCl}_{3}\right)$.

\section{General procedure for the synthesis of homoallylic alcohols 5-15.}

Scandium trifluoromethanesulfonate $(16 \mathrm{mg}, 0.03 \mathrm{mmol})$ was introduced in a $10-\mathrm{mL}$ round-bottom flask, which was then flushed with Ar. $\mathrm{CH}_{2} \mathrm{Cl}_{2}(1.5 \mathrm{~mL})$ was added and the mixture was cooled to $78^{\circ} \mathrm{C}$. The aldehyde $(0.33 \mathrm{mmol})$ was added, followed by a solution of boronate $4 \mathbf{a}(0.36 \mathrm{mmol})$ in $\mathrm{CH}_{2} \mathrm{Cl}_{2}(0.5 \mathrm{~mL})$ dropwise over 5 minutes. The resulting mixture was stirred at $-78^{\circ} \mathrm{C}$ for 16 hours, then DIBAL-H (1.0 M in toluene, $0.66 \mathrm{~mL}, 0.66 \mathrm{mmol})$ was added. The mixture was stirred at $-78^{\circ} \mathrm{C}$ for 30 minutes, then $1 \mathrm{~N} \mathrm{HCl}(5 \mathrm{~mL})$ was added and the flask was allowed to warm up to ambient temperature. The resulting layers were separated, then the aqueous layer was extracted with $\mathrm{Et}_{2} \mathrm{O}(3 \square 5 \mathrm{~mL})$. The combined organic layers were dried over $\mathrm{MgSO}_{4}$, filtered and concentrated. Flash chromatography afforded the pure homoallylic alcohol. The enantiomer of $2 \mathrm{a}$ was used for $\mathbf{1 2}$.

\section{(1R)-1-phenyl-3-buten-1-ol (5)}

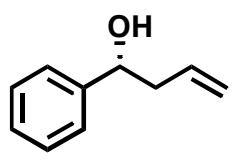

Flash chromatography (5\% EtOAc/hexanes) yielded a colourless oil (41 mg, 85\%), which gave satisfactory analytical data. ${ }^{9}$

TLC (15\% EtOAc/Hexane, PMA): 0.23;

HPLC : Chiralcel OD-RH, $40 \% i \mathrm{PrOH} / \mathrm{H}_{2} \mathrm{O}, 0.40 \mathrm{~mL} / \mathrm{min}$., UV detection at $210 \mathrm{~nm}$, , major peak at 22.3 min., minor peak at $25.7 \mathrm{~min} . .92 \%$ ee.

(3S)1-phenyl-5-hexen-3-ol (6)

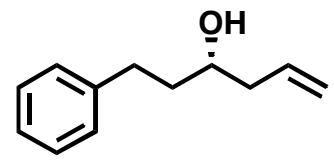

Flash chromatography (5\% EtOAc/hexanes) yielded a colourless oil (37 mg, 64\%), which gave satisfactory analytical data. ${ }^{9}$

TLC (15\% EtOAc/Hexane, PMA): 0.25;

HPLC : Chiralcel OD-RH, $40 \% i \mathrm{PrOH} / \mathrm{H}_{2} \mathrm{O}, 0.40 \mathrm{~mL} / \mathrm{min}$., UV detection at $210 \mathrm{~nm}$, , major peak at 40.2 min., minor peak at $49.4 \mathrm{~min}$. . $97 \%$ ee. 


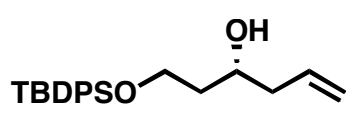

Flash chromatography (5\% EtOAc/hexanes) yielded a colourless oil (79mg, 86\%), which gave satisfactory analytical data. ${ }^{4}$

TLC (15\% EtOAc/ hexanes, PMA): 0.30;

HPLC : Chiralcel AD-RH, 45\% $i \mathrm{PrOH} / \mathrm{H}_{2} \mathrm{O}, 0.40 \mathrm{~mL} / \mathrm{min}$., UV detection at $210 \mathrm{~nm}$, major peak at 60.8 min., minor peak at $67.8 \mathrm{~min} . .93 \%$ ee.

\section{(2R)-1-(Benzyloxy)-4-penten-2-ol (8)}

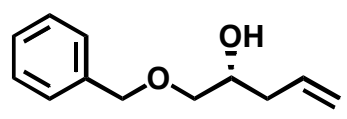

Flash chromatography (5\% EtOAc/Toluene) yielded a colourless oil (39 mg, 62\%), which gave satisfactory analytical data. ${ }^{4}$

TLC (15\% EtOAc/Toluene, PMA): 0.15;

HPLC : Chiralcel OD, 2\% $\mathrm{iPrOH} /$ hexane, $0.40 \mathrm{~mL} / \mathrm{min}$., UV detection at $210 \mathrm{~nm}$, major peak at 49.3 min., minor peak at 52.5 min.. $77 \%$ ee.

(2R)-1-(t-Butyldimethylsilyloxy)-4-penten-2-ol (9)

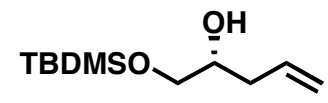

Flash chromatography (10\% EtOAc/hexanes) yielded a colourless oil (54 mg, 75\%), which gave satisfactory analytical data. ${ }^{4}$

TLC (15\% EtOAc/hexanes, PMA): 0.40;

${ }^{19} \mathrm{~F}$ NMR (376 MHz, $\mathrm{CDCl}_{3}$ ) $\square-71.90$ (Major), -71.99 (minor) 90\% ee on Mosher ester derivative.

\section{(3R)-1-(t-Butyldiphenylsilyloxy)-5-hexen-3-ol (10)}

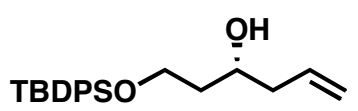

Flash chromatography (5\% EtOAc/Hexanes) yielded a colourless oil (69 mg, 61\%), which gave satisfactory analytical data. ${ }^{4}$

TLC (15\% EtOAc/hexanes, PMA): 0.25;

HPLC : Chiralcel AD-RH, 45\% $i \mathrm{PrOH} / \mathrm{H}_{2} \mathrm{O}, 0.33 \mathrm{~mL} / \mathrm{min}$., UV detection at $210 \mathrm{~nm}$, major peak at 75.1 min., minor peak at $88.4 \min . .90 \%$ ee.

\footnotetext{
${ }^{4}$ Roush, W.R.; Hoong, L.K.; Palmer, M.A.J.; Straub, J.A.; Palkowitz, A.D. J. Org. Chem. 1990, 55, 4117-4126.
} 


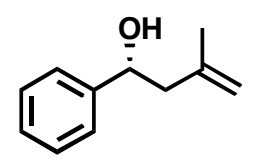

Flash chromatography (5\% EtOAc/hexanes) yielded a colourless oil (30 mg, 64\%), which gave satisfactory analytical data. ${ }^{9}$

TLC (15\% EtOAc/hexanes, PMA): 0.25;

HPLC : Chiralcel AD-RH, 50\% $i \mathrm{PrOH} / \mathrm{H}_{2} \mathrm{O}, 0.31 \mathrm{~mL} / \mathrm{min}$., UV detection at $210 \mathrm{~nm}$, major peak at 18.0 min., minor peak at $20.2 \min . .98 \%$ ee.

\section{(3R)-5-Methyl-1-phenyl-5-hexen-3-ol (12)}

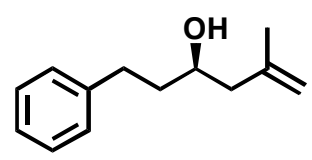

Flash chromatography (5\% EtOAc/hexanes) yielded a colourless oil (44 mg, 76\%), which gave satisfactory analytical data. ${ }^{9}$

TLC (15\% EtOAc/hexanes, PMA): 0.25;

HPLC : Chiralcel OD-RH, 45\% $i \mathrm{PrOH} / \mathrm{H}_{2} \mathrm{O}, 0.4 \mathrm{~mL} / \mathrm{min}$., UV detection at $210 \mathrm{~nm}$, minor peak at 35.0 min., major peak at 39.9 min.. $97 \%$ ee.

(3S)-1-(t-Butyldiphenylsilyloxy)-5-methyl-5-hexen-3-ol (13)

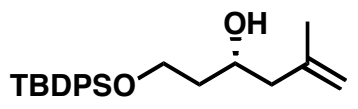

Flash chromatography (5\% EtOAc/hexanes) yielded a colourless oil (90 mg, 77\%), which gave satisfactory analytical data.

TLC (15\% EtOAc/hexanes, PMA): 0.30;

$[\square]_{\mathrm{D}}^{25}+0.89^{\circ}\left(c=1.23, \mathrm{CHCl}_{3}\right)$

${ }^{1} \mathrm{H}$ NMR (300 MHz, $\mathrm{CDCl}_{3}$ ): $\partial$ 7.70-7.67 (m, 4H), 7.44-7.39 (m, 6H), $4.84(\mathrm{dd}, J=3.7 \mathrm{~Hz}, 1.5 \mathrm{~Hz}, 1 \mathrm{H})$, $4.57(\mathrm{~d}, J=0.8 \mathrm{~Hz}, 1 \mathrm{H}), 4.09-4.03(\mathrm{~m}, 1 \mathrm{H}), 3.94-3.80(\mathrm{~m}, 2 \mathrm{H}) 2.96(\mathrm{~d}, J=2.4 \mathrm{~Hz}, 1 \mathrm{H}), 2.29-2.14(\mathrm{~m}$, $2 \mathrm{H}), 1.77(\mathrm{~s}, 3 \mathrm{H}), 1.76-1.68(\mathrm{~m}, 2 \mathrm{H}), 1.06(\mathrm{~s}, 9 \mathrm{H})$;

${ }^{13} \mathrm{C}$ NMR $\left(125 \mathrm{MHz}, \mathrm{CDCl}_{3}\right): \partial 142.7,135.5,135.5,133.2,133.1129 .7,127.7,112.9,68.6,62.9,46.0$, $38.3,26.8,22.5,19.1$;

IR $\left(\mathrm{CH}_{2} \mathrm{Cl}_{2}\right.$ cast, $\left.\mathrm{cm}^{-1}\right): 3455,3049,29.31,1472,1427,1111$;

HRMS (EI, $m / z$ ) Calcd for $\mathrm{C}_{23} \mathrm{H}_{32} \mathrm{O}_{2} \mathrm{NaSi}$ : 391.20638. Found: 391.20635;

${ }^{19} \mathrm{~F}$ NMR (376 MHz, $\mathrm{CDCl}_{3}$ ) $\square-71.69$ (Major), -71.79 (minor) 97\% ee on Mosher ester derivative. 


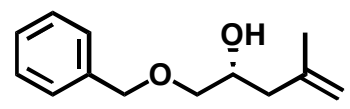

Flash chromatography (5\% EtOAc/toluene) yielded a colourless oil (46 mg, 70\%).

TLC (15\% EtOAc/hexanes, PMA): 0.15;

$[\square]^{25}-1.86^{\circ}\left(c=1.30, \mathrm{CHCl}_{3}\right)$

${ }^{1} \mathrm{H}$ NMR $\left(300 \mathrm{MHz}, \mathrm{CDCl}_{3}\right):$ 2 7.36-7.26 (m, 5H), $4.83(\mathrm{dd}, J=1.7 \mathrm{~Hz}, 1.7 \mathrm{~Hz}, 1 \mathrm{H}), 4.76($,dd, $J=1.0$ $\mathrm{Hz}, 1.0 \mathrm{~Hz}, 1 \mathrm{H}), 4.55$ (s, 2H), 3.99-3.95 (m, 1H), 3.50 (,dd, $J=9.5 \mathrm{~Hz}, 3.5 \mathrm{~Hz}, 1 \mathrm{H}), 3.37$ (,dd, $J^{l}=9.5 J^{2}$ $7.1 \mathrm{~Hz}, 1 \mathrm{H}), 2.27(\mathrm{~d}, J=2.9 \mathrm{~Hz}, 1 \mathrm{H}), 2.22-2.16(\mathrm{~m}, 2 \mathrm{H}), 1.75(\mathrm{~s}, 3 \mathrm{H})$;

${ }^{13} \mathrm{C}$ NMR $\left(125 \mathrm{MHz}, \mathrm{CDCl}_{3}\right): \partial$ 142.0, 138.0, 128.4, 127.7, 127.7,127.7, 113.2, 74.2, 73.4, 68.2, 41.9, 22.5

IR $\left(\mathrm{CH}_{2} \mathrm{Cl}_{2}\right.$ cast, $\left.\mathrm{cm}^{-1}\right): 3445,3070,3030,2913,1646,1453,1099$;

MS (ES, $m / z) 229.1(\mathrm{M}+\mathrm{Na})$;

HPLC : Chiralcel OD-RH, 65\% iPrOH/ $\mathrm{H}_{2} \mathrm{O}, 0.45 \mathrm{~mL} / \mathrm{min}$, UV detection at $210 \mathrm{~nm}$, Minor peak at 52.7 min., major peak at $55.9 \min . .97 \%$ ee.

(2R)-1-(t-Butyldimethylsilyloxy)-4-methyl-4-penten-2-ol (15)

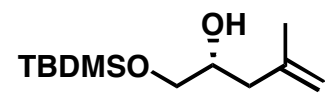

Flash chromatography (10\% EtOAc/hexanes) yielded a colourless oil (38 mg, 58\%).

TLC (15\% EtOAc/Toluene, PMA): 0.40;

$[\square]_{\mathrm{D}}^{25}-1.86^{\circ}\left(c=1.30, \mathrm{CHCl}_{3}\right)$

${ }^{1} \mathrm{H}$ NMR $\left(300 \mathrm{MHz}, \mathrm{CDCl}_{3}\right): \partial 4.81(\mathrm{dd}, J=1.4 \mathrm{~Hz}, 1.4 \mathrm{~Hz}, 1 \mathrm{H}), 4.76(\mathrm{dd}, J=2.0 \mathrm{~Hz}, 1.0 \mathrm{~Hz}, 1 \mathrm{H}), 3.81-$

$3.77(\mathrm{~m}, 1 \mathrm{H}), 3.60(\mathrm{dddd}, J=10.0 \mathrm{~Hz}, 4.8 \mathrm{~Hz}, 3.9 \mathrm{~Hz}, 0.9 \mathrm{~Hz}, 1 \mathrm{H}), 3.45$ (dd, $J=10.0 \mathrm{~Hz}, 7.0 \mathrm{~Hz}, 1 \mathrm{H})$,

$2.33(\mathrm{~d}, J=2.9 \mathrm{~Hz}, 1 \mathrm{H}), 2.15(\mathrm{~d}, J=6.8 \mathrm{~Hz}, 2 \mathrm{H}), 1.75(\mathrm{~s}, 3 \mathrm{H}), 0.89(\mathrm{~s}, 9 \mathrm{H}), 0.06(\mathrm{~s}, 6 \mathrm{H})$;

${ }^{13} \mathrm{C}$ NMR $\left(125 \mathrm{MHz}, \mathrm{CDCl}_{3}\right): \partial$ 142.3, 112.9, 69.7, 66.9, 41.7, 26.0, 22.7, 18.4, -5.1, -5.2;

IR $\left(\mathrm{CH}_{2} \mathrm{Cl}_{2}\right.$ cast, $\left.\mathrm{cm}^{-1}\right): 3467,3076,2955,2929,1648,1472,1361,1120$;

${ }^{19} \mathrm{~F}$ NMR (376 MHz, $\mathrm{CDCl}_{3}$ ) $\square-71.91$ (Major), -71.99 (minor) 95\% ee on Mosher ester derivative.

\section{General procedure for the synthesis of homoallylic alcohols 16-19.}

Scandium trifluoromethanesulfonate $(16 \mathrm{mg}, 0.03 \mathrm{mmol})$ was introduced in a $10-\mathrm{mL}$ round-bottom flask, which was then flushed with Ar. $\mathrm{CH}_{2} \mathrm{Cl}_{2}(0.3 \mathrm{~mL})$ was added and the mixture was cooled to $78^{\circ} \mathrm{C}$. The aldehyde $(0.45 \mathrm{mmol})$ was added, followed by a solution of boronate $3 \mathbf{a}(0.3 \mathrm{mmol})$ in $\mathrm{CH}_{2} \mathrm{Cl}_{2}(0.3 \mathrm{~mL})$ dropwise over 5 minutes. The resulting mixture was stirred at $-78^{\circ} \mathrm{C}$ for 24 hours, then 
DIBAL-H (1.0 M in toluene, $0.67 \mathrm{~mL}, 0.67 \mathrm{mmol})$ was added. The mixture was stirred at $-78^{\circ} \mathrm{C}$ for 60 minutes, then $1 \mathrm{~N} \mathrm{HCl}(5 \mathrm{~mL})$ was added and the flask was allowed to warm up to ambient temperature. The resulting layers were separated, then the aqueous layer was extracted with $\mathrm{Et}_{2} \mathrm{O}(3 \square 5 \mathrm{~mL})$. The combined organic layers were dried over $\mathrm{MgSO}_{4}$, filtered and concentrated. Flash chromatography afforded the pure homoallylic alcohol.

\section{$(1 R, 2 R)-2-M e t h y l-1-p h e n y l-3-b u t e n-1-o l ~(16)$}

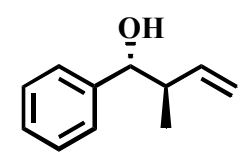

Flash chromatography (2.5\% EtOAc/Hexane) gave the pure product as a colourless oil (60\% yield), which gave satisfactory analytical data. ${ }^{5}$

$[\square]_{D}^{25}=+92.0^{\circ}\left(c=1.62, \mathrm{CHCl}_{3}\right.$, lit. $^{2}-73.4^{\circ}(66 \% \mathrm{ee})$ for the enantiomer $)$

HPLC : Chiralcel OD-RH, 30\% $i \mathrm{PrOH} / \mathrm{H}_{2} \mathrm{O}, 0.45 \mathrm{~mL} / \mathrm{min}$., UV detection at $210 \mathrm{~nm}$, major peak at $106.8 \mathrm{~min}$. , minor peak at $118 \mathrm{~min} ., 97 \%$ ee.

\section{(3S, 4R)-4-Methyl-1-phenyl-5-hexen-3-ol (17)}

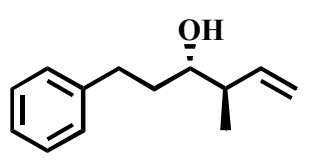

Flash chromatography (5\% EtOAc/Hexane) gave the pure product as a colourless oil (71\% yield), which gave satisfactory analytical data. ${ }^{2}$ $[\square]_{\mathrm{D}}^{25}=-14.7^{\circ}\left(c=1.42, \mathrm{CHCl}_{3}\right.$, lit.: $^{2}+13.8^{\circ}$ (86\%ee $)$ for the enantiomer $)$

HPLC : Chiralcel AD-RH, 40\% $i \mathrm{PrOH} / \mathrm{H}_{2} \mathrm{O}, 0.33 \mathrm{~mL} / \mathrm{min}$., UV detection at $210 \mathrm{~nm}$, major peak at 64.8 min., minor peak at $73.3 \mathrm{~min} ., 96 \%$ ee.

\section{(3S, 4R)-1-(t-Butyldiphenylsilyloxy)-4-methyl-5-hexen-3-ol (18)}

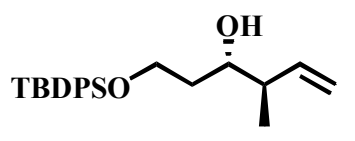

Flash chromatography (2.5\% EtOAc/Hexane) gave the pure product as a colourless oil (63\% yield), which gave satisfactory analytical data. ${ }^{6}$ $[\square]_{D}^{25}=+4.4^{\mathrm{o}}\left(c=1.46, \mathrm{CHCl}_{3}\right.$, lit.: ${ }^{6}+3.0$ (mixture of diastereomers $)$ )

\footnotetext{
${ }^{5}$ Tokuda, M.; Satoh, S.; Suginome, H. J.Org.Chem. 1989, 54, 5608-5613.

${ }^{6}$ Chemler, S.R.; Roush, W.R. J. Org. Chem. 1998, 63, 3800-3801.
} 
HPLC : Chiralcel AD-RH, 55\% $i \mathrm{PrOH} / \mathrm{H}_{2} \mathrm{O}, 0.31 \mathrm{~mL} / \mathrm{min}$., UV detection at $210 \mathrm{~nm}$, minor peak at 27.7 min., major peak at $31.9 \mathrm{~min} ., 94 \%$ ee.

$(2 R, 3 R)-1-(t$-Butyldimethylsilyloxy)-3-methyl-4-penten-2-ol (19)

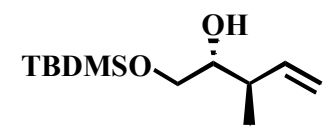

Flash chromatography (5\% EtOAc/hexanes) gave the pure product as a colourless oil (63\% yield), which gave satisfactory analytical data. ${ }^{7}$

${ }^{1} \mathrm{H}$ NMR (300 MHz, $\left.\mathrm{CDCl}_{3}\right):$ d $5.84(\mathrm{~m}, 1 \mathrm{H})$, 5.06-5.02 (m, 2H), 3.63-3.60 (m, 1H) 3.50-3.47 (m, 2H), $2.35(\mathrm{~d}, J=2 \mathrm{~Hz}, 1 \mathrm{H}), 2.30$ (apparent sextet, $J=7.0 \mathrm{~Hz}, 1 \mathrm{H}), 1.02(\mathrm{~d}, J=6.8 \mathrm{~Hz}, 3 \mathrm{H}), 0.88(\mathrm{~s}, 9 \mathrm{H}), 0.05$ $(\mathrm{s}, 6 \mathrm{H})$;

${ }^{13} \mathrm{C}$ NMR (125 MHz, $\left.\mathrm{CDCl}_{3}\right): \partial$ 140.3, 115.0, 74.8, 65.2, 41.4, 25.8, 18.2, 16.1, -5.4;

HRMS (EI, $m / z)$ Calcd for $\left(\mathrm{C}_{12} \mathrm{H}_{26} \mathrm{O}_{2} \mathrm{Si}-t\right.$-Bu): 173.09978. Found: 173.09970.

$[\square]_{\mathrm{D}}^{25}=+2.8^{\circ}\left(c=0.73 \mathrm{~g} / \mathrm{mL}, \mathrm{CHCl}_{3}\right)$.

HPLC (performed on the carbamate product from the reaction with phenylisocyanate) : Chiralcel OD, $2 \% \mathrm{iPrOH} / \mathrm{hexane}, 0.30 \mathrm{~mL} / \mathrm{min}$., $\mathrm{UV}$ detection at $210 \mathrm{~nm}$, major peak at $26.2 \mathrm{~min}$., minor peak at 24.8 $\min ., 96 \%$ ee.

\section{General procedure for the synthesis of syn-homoallylic alcohols 20-23.}

Scandium trifluoromethanesulfonate $(16 \mathrm{mg}, 0.03 \mathrm{mmol})$ and $4 \AA$ molecular sieves $(33 \mathrm{mg})$ were introduced in a $10-\mathrm{mL}$ round-bottom flask, which was then flushed with $\mathrm{Ar}$. $\mathrm{CH}_{2} \mathrm{Cl}_{2}(0.3 \mathrm{~mL})$ was added and the mixture was cooled to $-78^{\circ} \mathrm{C}$. The aldehyde $(0.50 \mathrm{mmol})$ was added, followed by a solution of boronate $4 \mathbf{a}(0.33 \mathrm{mmol})$ in $\mathrm{CH}_{2} \mathrm{Cl}_{2}(0.3 \mathrm{~mL})$ dropwise over 5 minutes. The resulting mixture was stirred at $-78^{\circ} \mathrm{C}$ for 24 hours, then DIBAL-H (1.0 M in toluene, $\left.0.66 \mathrm{~mL}, 0.66 \mathrm{mmol}\right)$ was added. The mixture was stirred at $-78^{\circ} \mathrm{C}$ for 30 minutes, then $1 \mathrm{~N} \mathrm{HCl}(5 \mathrm{~mL})$ was added and the flask was allowed to warm up to ambient temperature. The resulting layers were separated, then the aqueous layer was extracted with EtOAc $(3 \square 5 \mathrm{~mL})$. The combined organic layers were dried over $\mathrm{MgSO}_{4}$, filtered and concentrated. Flash chromatography afforded the pure homoallylic alcohol.

$(1 R, 2 S)-2-M e t h y l-1-p h e n y l-3-b u t e n-1-o l(20)$

7 Wipf, P.; Reeves, J.T. Chem. Commun.; 2002, 2066-2067. 


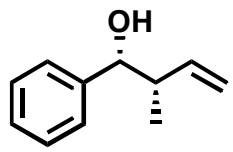

Flash chromatography (5\% EtOAc/Toluene) yielded a colourless oil (28.5 mg, 53\%), which gave satisfactory analytical data. ${ }^{8}$

TLC (10\% EtOAc/toluene, PMA): 0.23;

$[\square]^{25}+12.0^{\circ}\left(c=1.47, \mathrm{CHCl}_{3}\right.$, lit.: $.^{2,8}-15.0^{\circ}$ for the enantiomer, $\left.+15.2^{\circ}\right)$

HPLC : Chiralcel OD-RH, 35\% $i \mathrm{PrOH} / \mathrm{H}_{2} \mathrm{O}, 0.40 \mathrm{~mL} / \mathrm{min}$., UV detection at $210 \mathrm{~nm}$, major peak at 61.5 min., minor peak at $68.7 \mathrm{~min} ., 59 \%$ ee.

\section{(3S, 4S)-4-Methyl-1-phenyl-5-hexen-3-ol (21)}

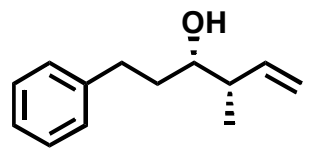

Flash chromatography (5\% EtOAc/Toluene) yielded a colourless oil (32 mg, 51\%), which gave satisfactory analytical data. ${ }^{9}$

TLC (10\% EtOAc/toluene, PMA): 0.35;

$[\square]_{D}^{25}-32.5^{\circ}\left(c=0.80, \mathrm{CHCl}_{3}\right)$.

HPLC : Chiralcel AD-RH, $40 \% i \mathrm{PrOH} / \mathrm{H}_{2} \mathrm{O}, 0.33 \mathrm{~mL} / \mathrm{min}$., UV detection at $210 \mathrm{~nm}$, major peak at 53.9 min., minor peak at $60.0 \mathrm{~min} ., 96 \%$ ee.

\section{(3S, 4S)-1-(t-Butyldiphenylsilyloxy)-4-methyl-5-hexen-3-ol (22)}

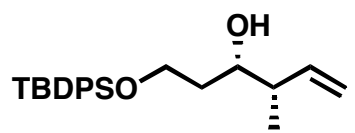

Flash chromatography (5\% EtOAc/Toluene) yielded a colourless oil (69.5 mg, 57\%), which gave satisfactory analytical data. ${ }^{10,11}$

TLC (5\% EtOAc/toluene, PMA): 0.29;

$[\square]_{\mathrm{D}}^{25}-4.4^{\mathrm{o}}\left(c=1.89, \mathrm{CHCl}_{3}\right.$, lit.: ${ }^{10,11} .+3.3^{\mathrm{o}},+5.5^{\circ}$ for the enantiomer $)$

HPLC : Chiralcel AD-RH, 55\% $\mathrm{iPrOH} / \mathrm{H}_{2} \mathrm{O}, 0.30 \mathrm{~mL} / \mathrm{min}$., UV detection at $210 \mathrm{~nm}$, minor peak at 24.6 min., major peak at 28.2 min., $96 \%$ ee.

\footnotetext{
${ }^{8}$ Iseki, K.; Kuroki, Y.; Takahashi, M.; Kishimoto, S.; Kobayashi, Y. Tetrahedron 1997, 53, 3513-3526.

${ }^{9}$ Kobayashi, S.; Nishio, K. J. Org. Chem. 1994, 59, 6620-6628.

10 Boeckman, R.K.; Charette, A.B.; Asberom, T.; Johnston, B.H. J. Am. Chem. Soc. 1991, 113, 5337-5353.
} 
$(2 R, 3 S)-1-(t$-Butyldimethylsilyloxy)-3-methyl-4-penten-2-ol (23)

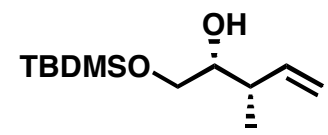

Flash chromatography (5\% EtOAc/hexanes) gave the pure product as a colourless oil (43 mg, 57\%). TLC (5\% EtOAc/toluene, PMA): 0.20;

${ }^{1} \mathrm{H}$ NMR (300 MHz, $\mathrm{CDCl}_{3}$ ): $\partial 5.70$ (ddd, $\left.J=18.3,10.3,8.0 \mathrm{~Hz}, 1 \mathrm{H}\right)$, 5.06-4.97 (m, 2H), 3.66-3.60 (m, 1H) $3.48-3.39(\mathrm{~m}, 2 \mathrm{H}), 2.44(\mathrm{~d}, J=3.7 \mathrm{~Hz}, 1 \mathrm{H}), 2.26$ (apparent sextet, $J=7.0 \mathrm{~Hz}, 1 \mathrm{H}), 1.07(\mathrm{~d}, J=6.8$ $\mathrm{Hz}, 3 \mathrm{H}), 0.88$ (s, 9H), 0.05 (s, 6H);

${ }^{13} \mathrm{C}$ NMR (125 MHz, $\left.\mathrm{CDCl}_{3}\right): \partial$ 140.5, 114.9, 74.7, 65.2, 41.0, 25.8, 18.2, 16.0, -5.4;

IR $\left(\mathrm{CH}_{2} \mathrm{Cl}_{2}\right.$ cast, $\left.\mathrm{cm}^{-1}\right): 3574,3482,3078,2956,2929,2857,1640,1463,1257,1100,837$;

HRMS (EI, $m / z$ ) Calcd for $\mathrm{C}_{12} \mathrm{H}_{26} \mathrm{O}_{2} \mathrm{NaSi}: 253.15943$. Found: 253.15936.

$[\square]_{\mathrm{D}}^{25}-15.9^{\circ}\left(c=0.83, \mathrm{CHCl}_{3}\right)$.

HPLC (performed on the carbamate product from the reaction with phenylisocyanate) : Chiralcel OD, $2 \% \mathrm{iPrOH} /$ hexane, $0.30 \mathrm{~mL} / \mathrm{min}$., UV detection at $210 \mathrm{~nm}$, major peak at $23.3 \mathrm{~min}$., minor peak at 24.3 $\min ., 96 \%$ ee.

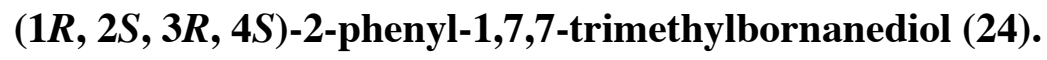

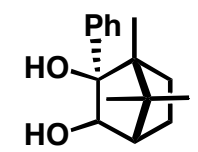

This compound was prepared according to published procedures. ${ }^{3}$

11 Scarlato, G.R.; DeMattei, J.A.; Chong, L.S.; Ogawa, A.K.; Lin, M.R.; Armstrong, R.W. J. Org. Chem. 1996, 61, 61396152. 


\section{Copies of ${ }^{1} \mathrm{H}$ and ${ }^{15} \mathrm{C}$ NMR spectra}

$(1 R, 2 S, 3 R, 4 S)$-2,3-O-[Allylboryl]-2-phenyl-1,7,7-trimethylbornanediol (1a)

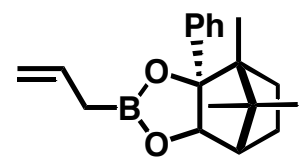

${ }^{1} \mathrm{H}$ and APT ${ }^{13} \mathrm{C}$ NMR
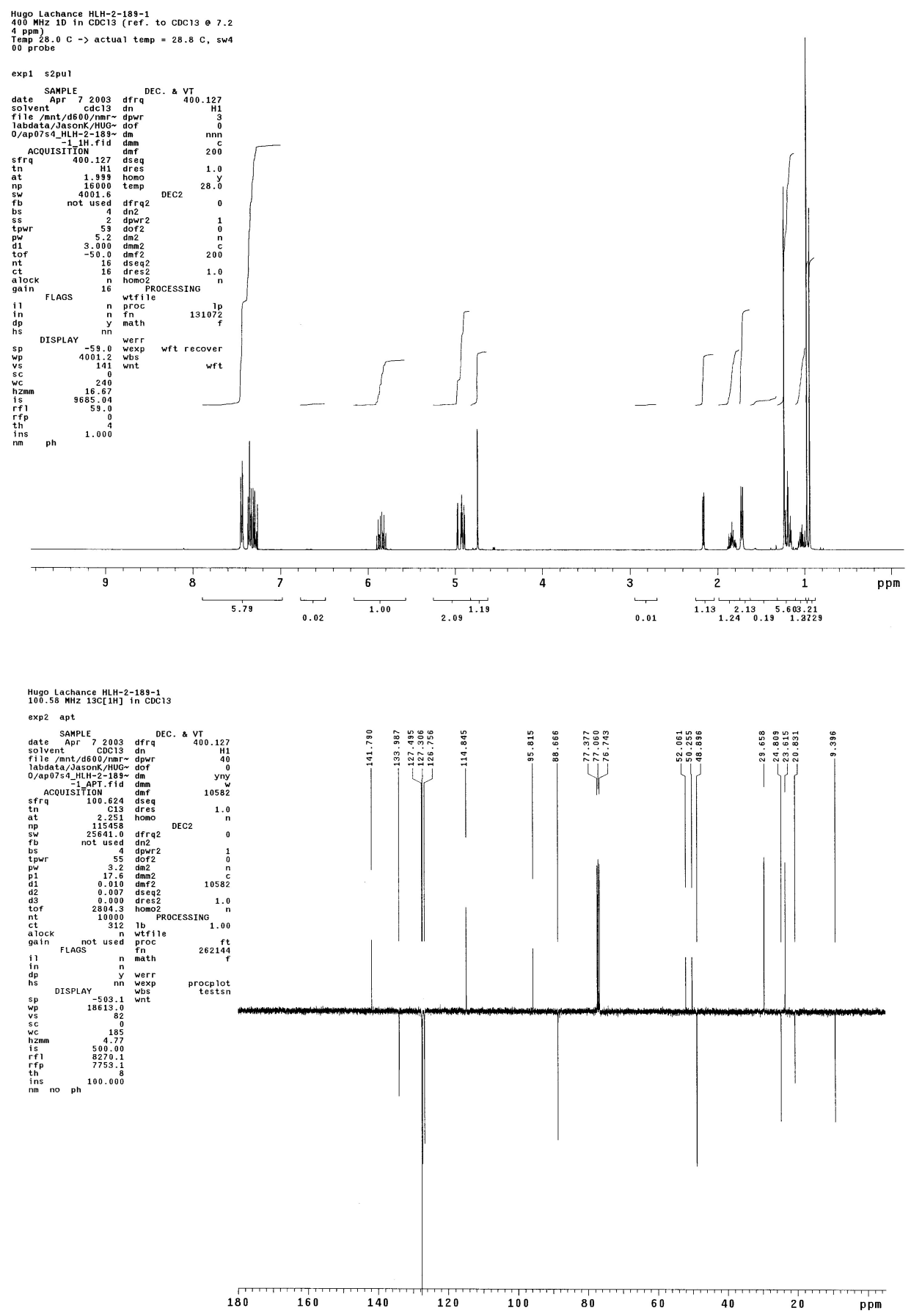
$(1 R, 2 S, 3 R, 4 S)$-2,3-O-[2-methylallyl-3-boryl]-2-phenyl-1,7,7-trimethylbornanediol (2a)

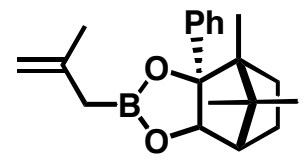

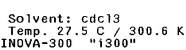

${ }^{1} \mathrm{H}$ and APT ${ }^{13} \mathrm{C}$ NMR

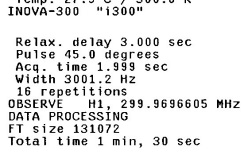

Total time 1 min, $30 \mathrm{sec}$
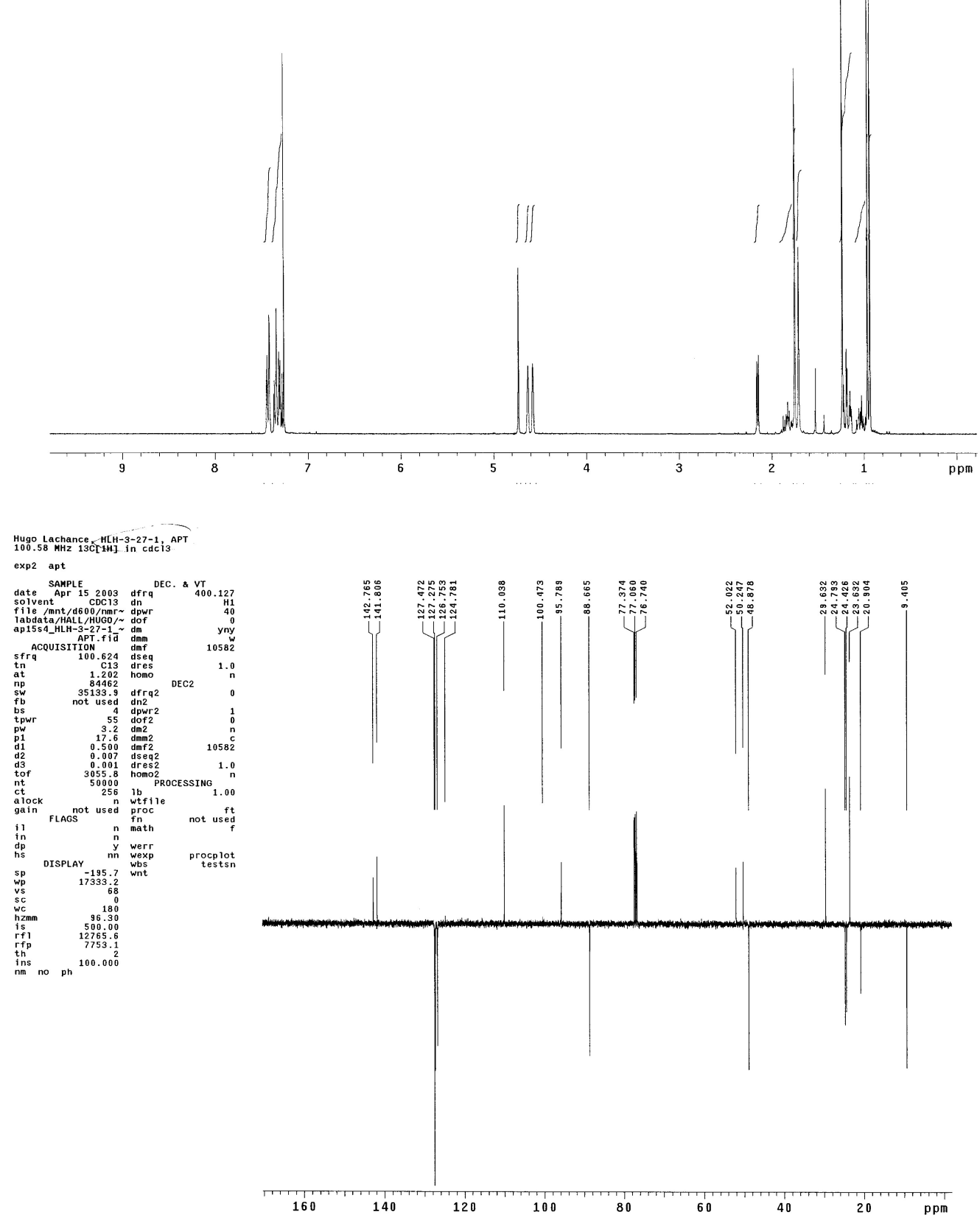
$(1 R, 2 S, 3 R, 4 S)$-2,3-O-[(E)-2-butenylboryl]-2-phenyl-1,7,7-trimethylbornanediol (3a)
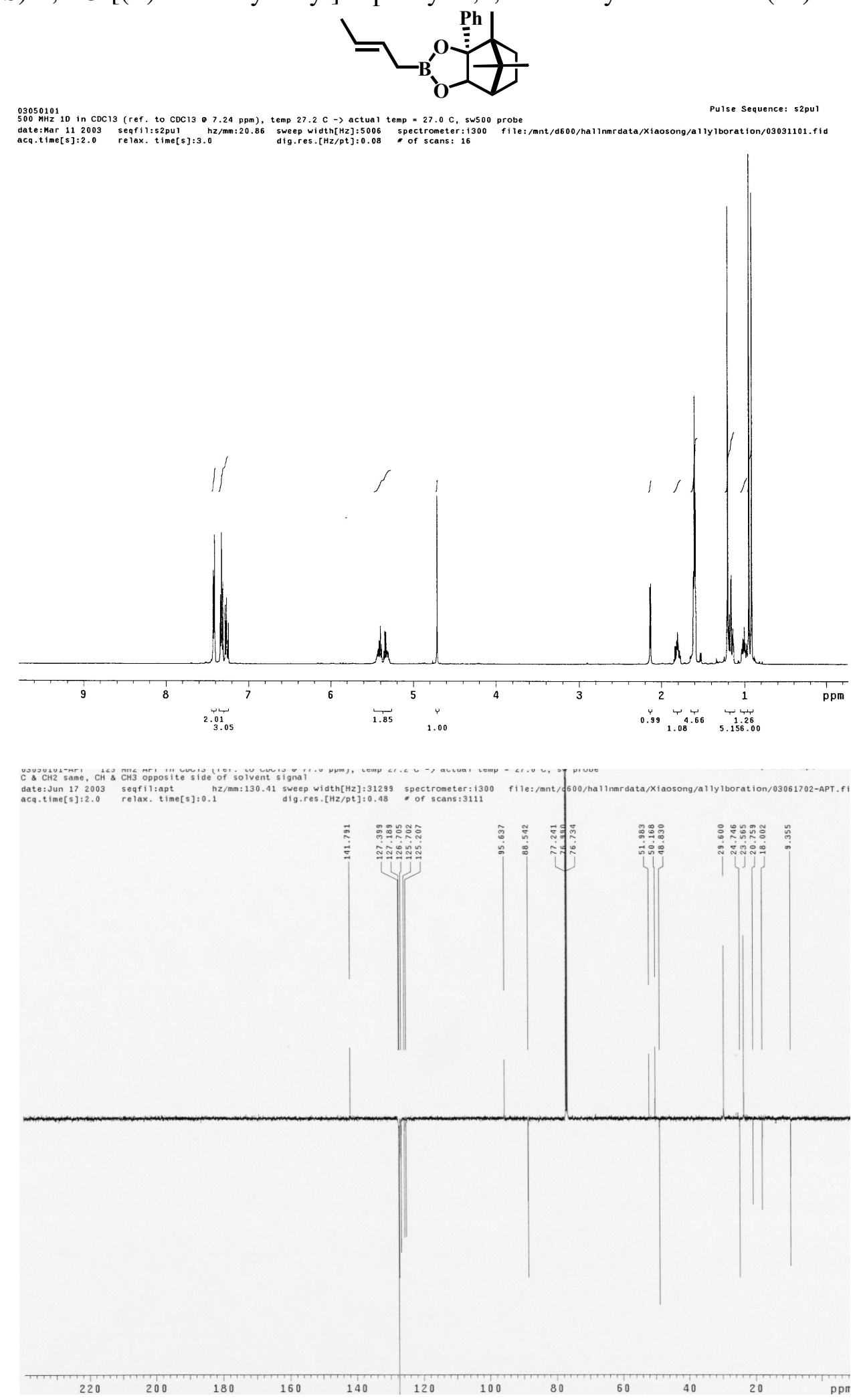
$(1 R, 2 S, 3 R, 4 S)$-2,3-O-[(Z)-2-butenylboryl]-2-phenyl-1,7,7-trimethylbornanediol (4a)
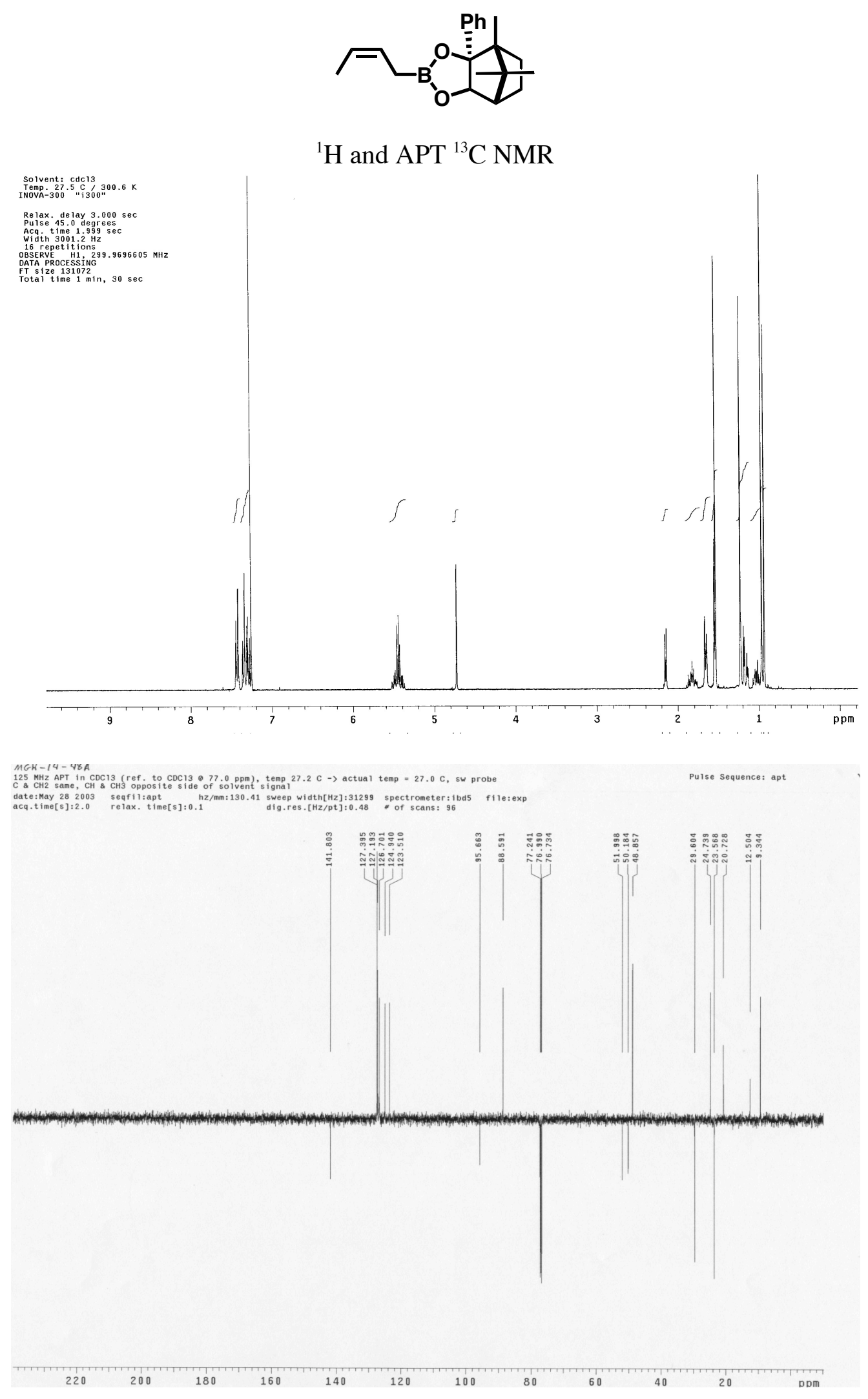
(1R)-1-phenyl-3-buten-1-ol (5)

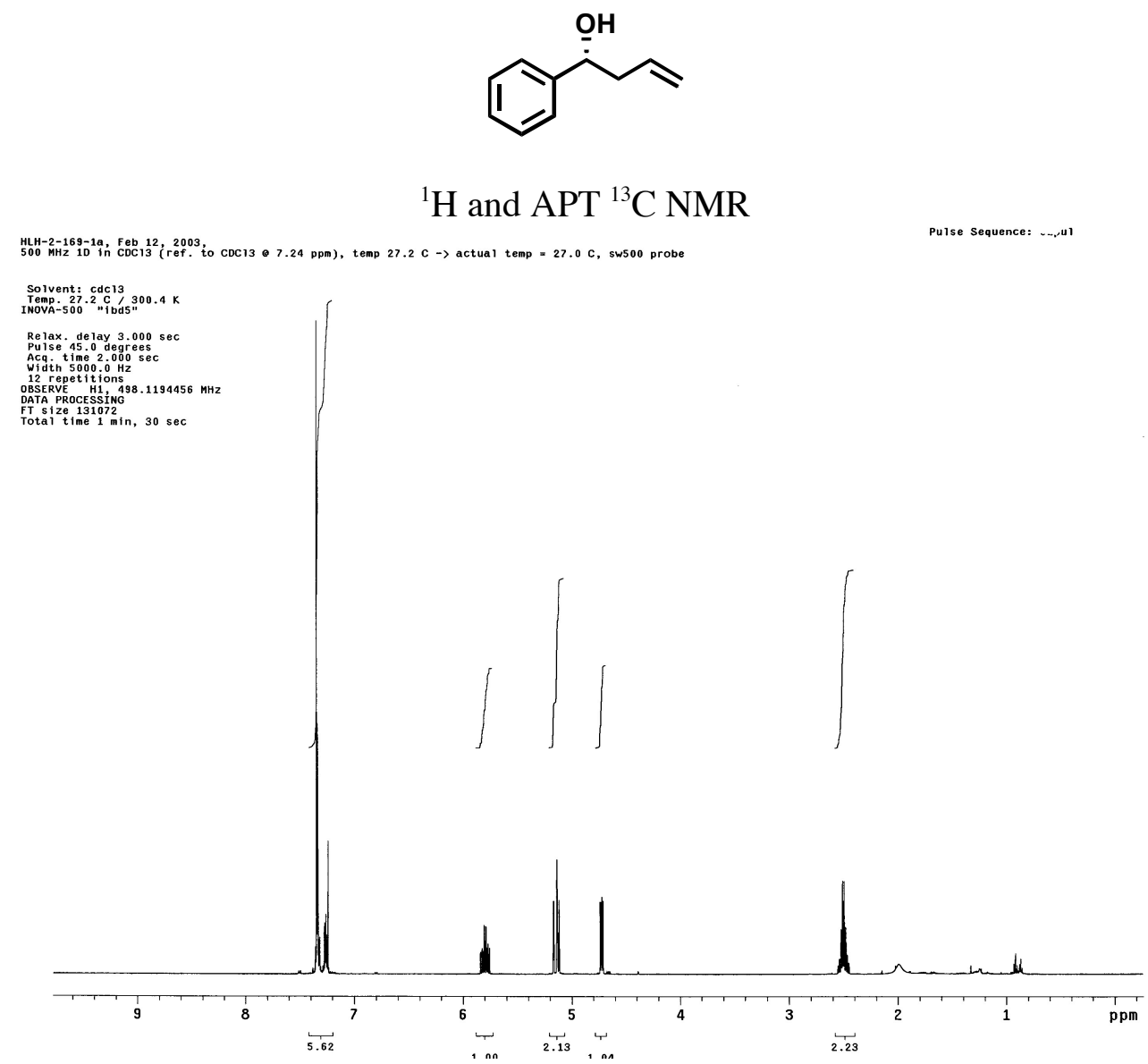

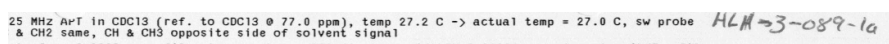

Pulse Sequence: apt

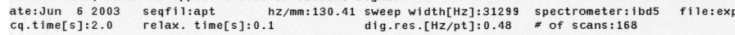

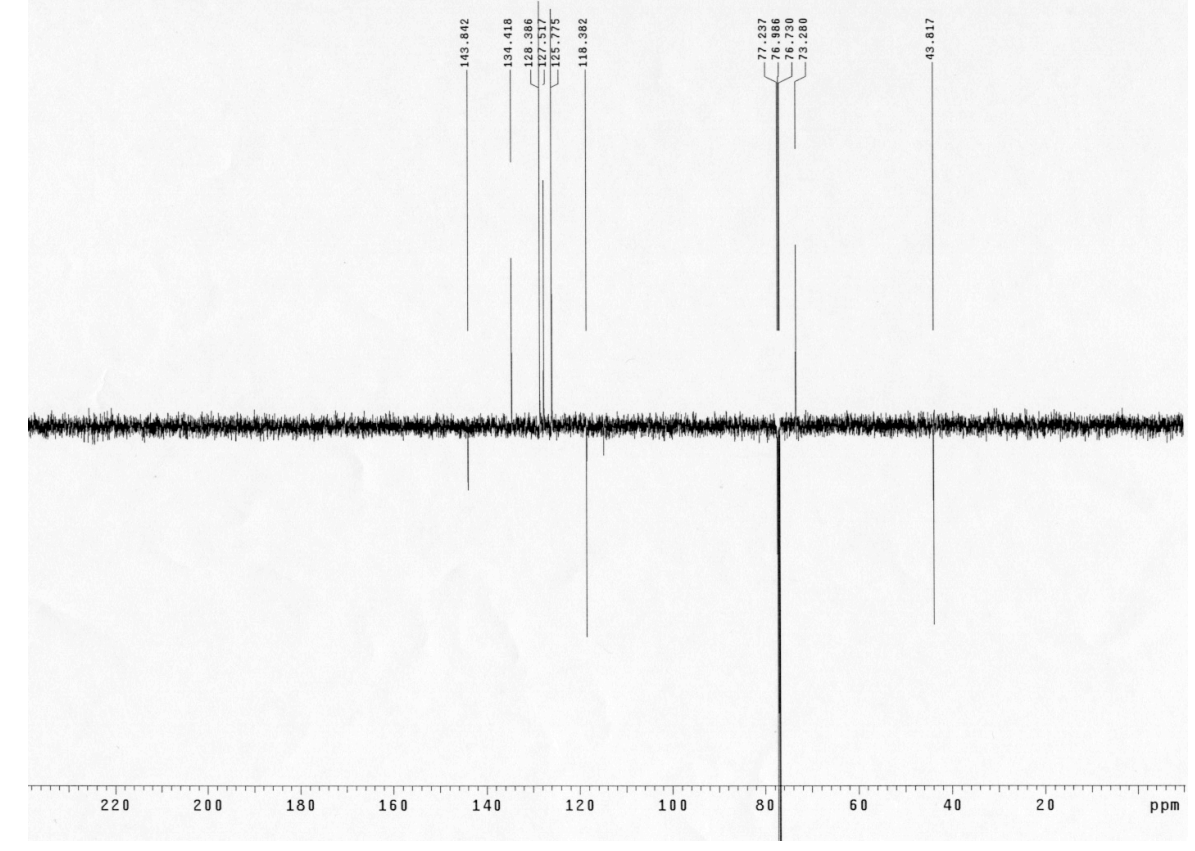


(3S)1-phenyl-5-hexen-3-ol (6)

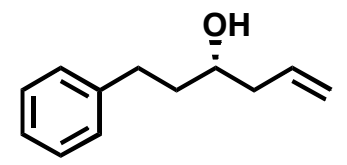

${ }^{1} \mathrm{H}$ and APT ${ }^{13} \mathrm{C}$ NMR
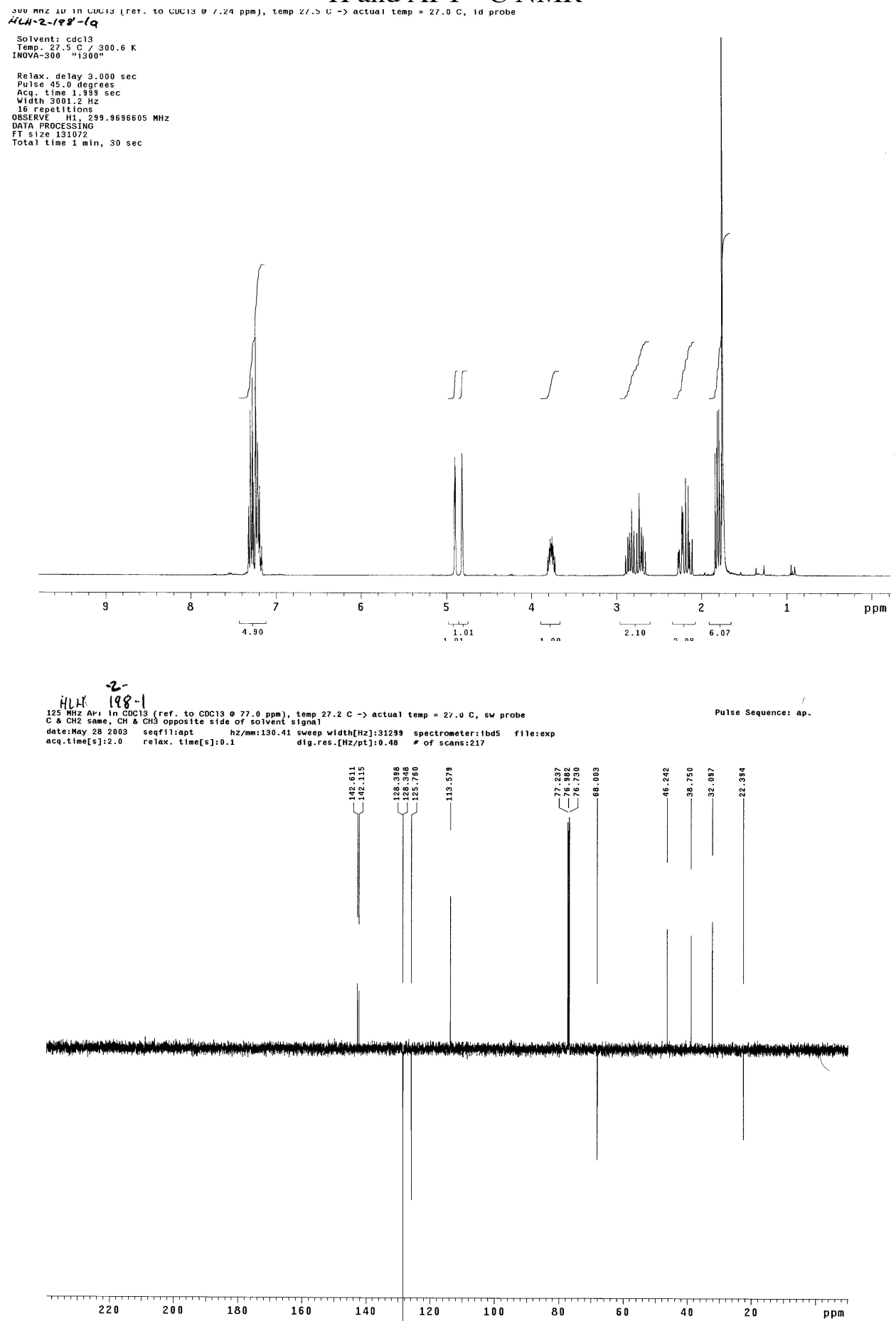
(3R)-1-( $t$-Butyldiphenylsilyloxy)-5-hexen-3-ol (7)
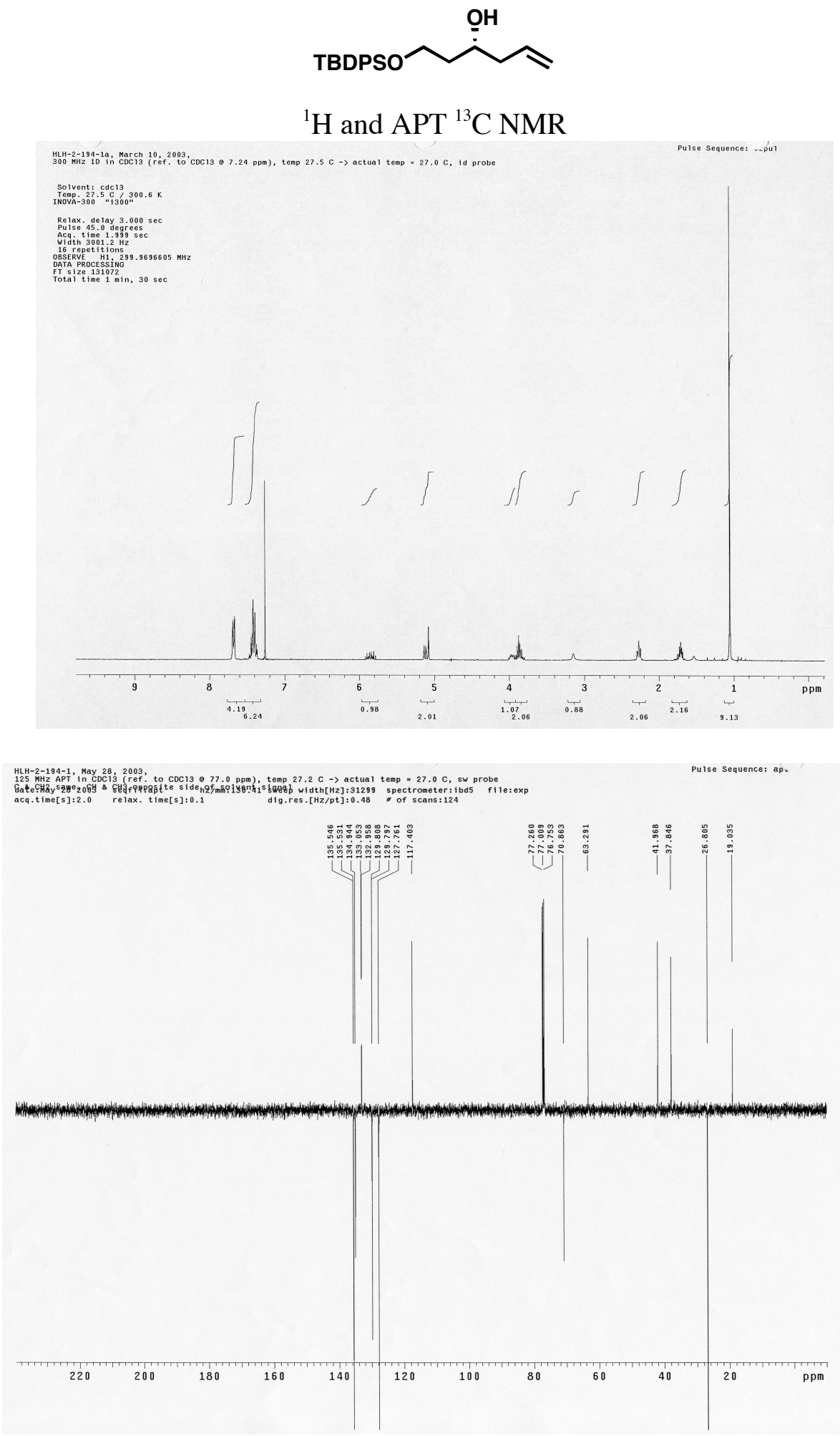
(2R)-1-(Benzyloxy)-4-penten-2-ol (8)

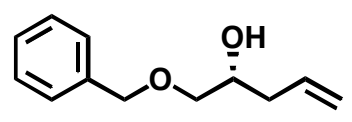

${ }^{1} \mathrm{H}$ and APT ${ }^{13} \mathrm{C}$ NMR
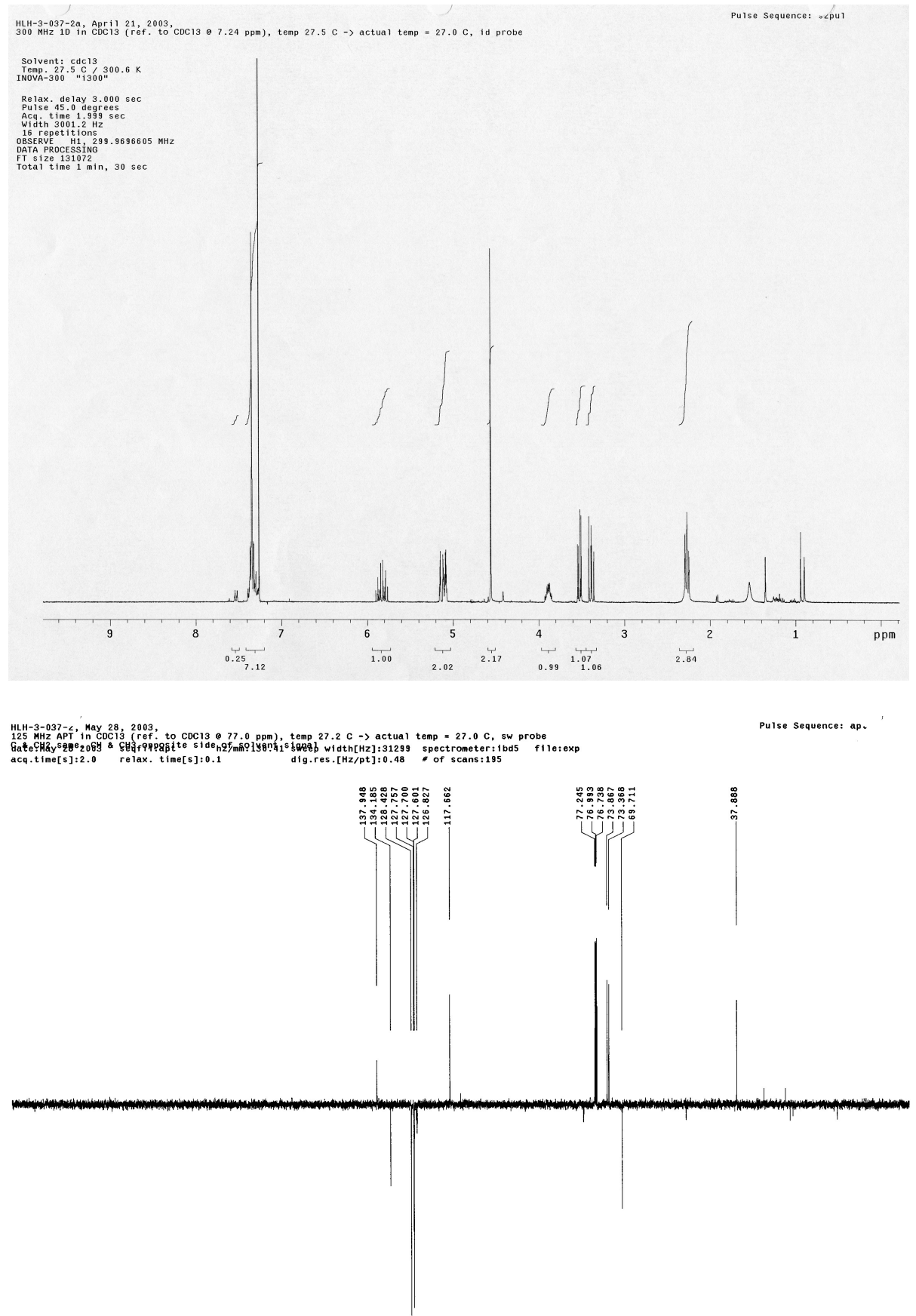

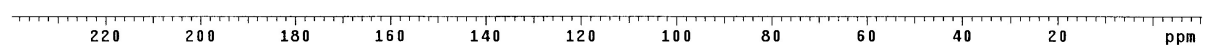


(2R)-1-( $t$-Butyldimethylsilyloxy)-4-penten-2-ol (9)

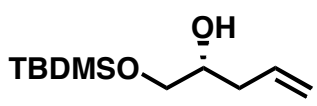

${ }^{1} \mathrm{H}$ NMR and APT ${ }^{13} \mathrm{C}$ NMR
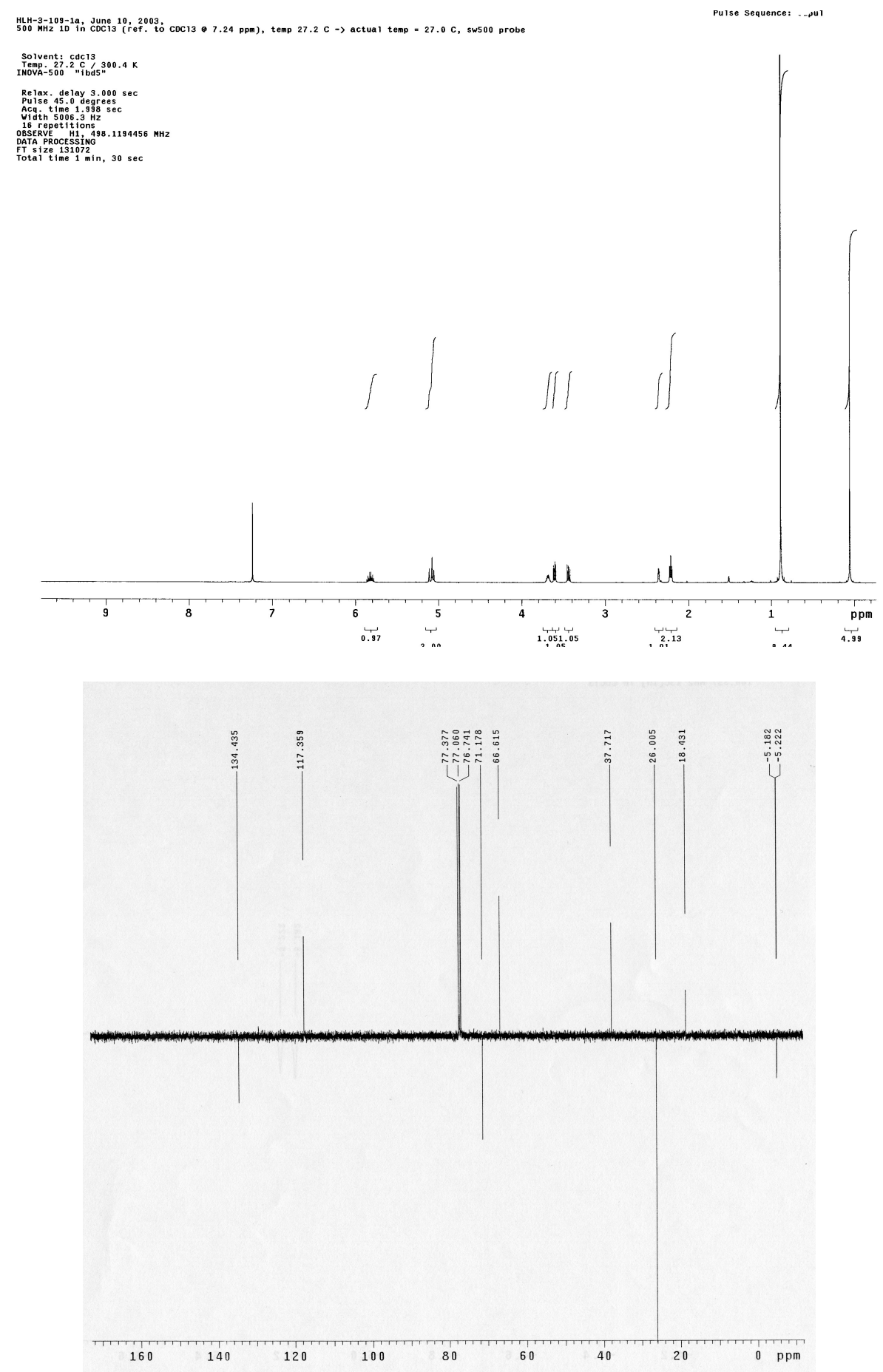

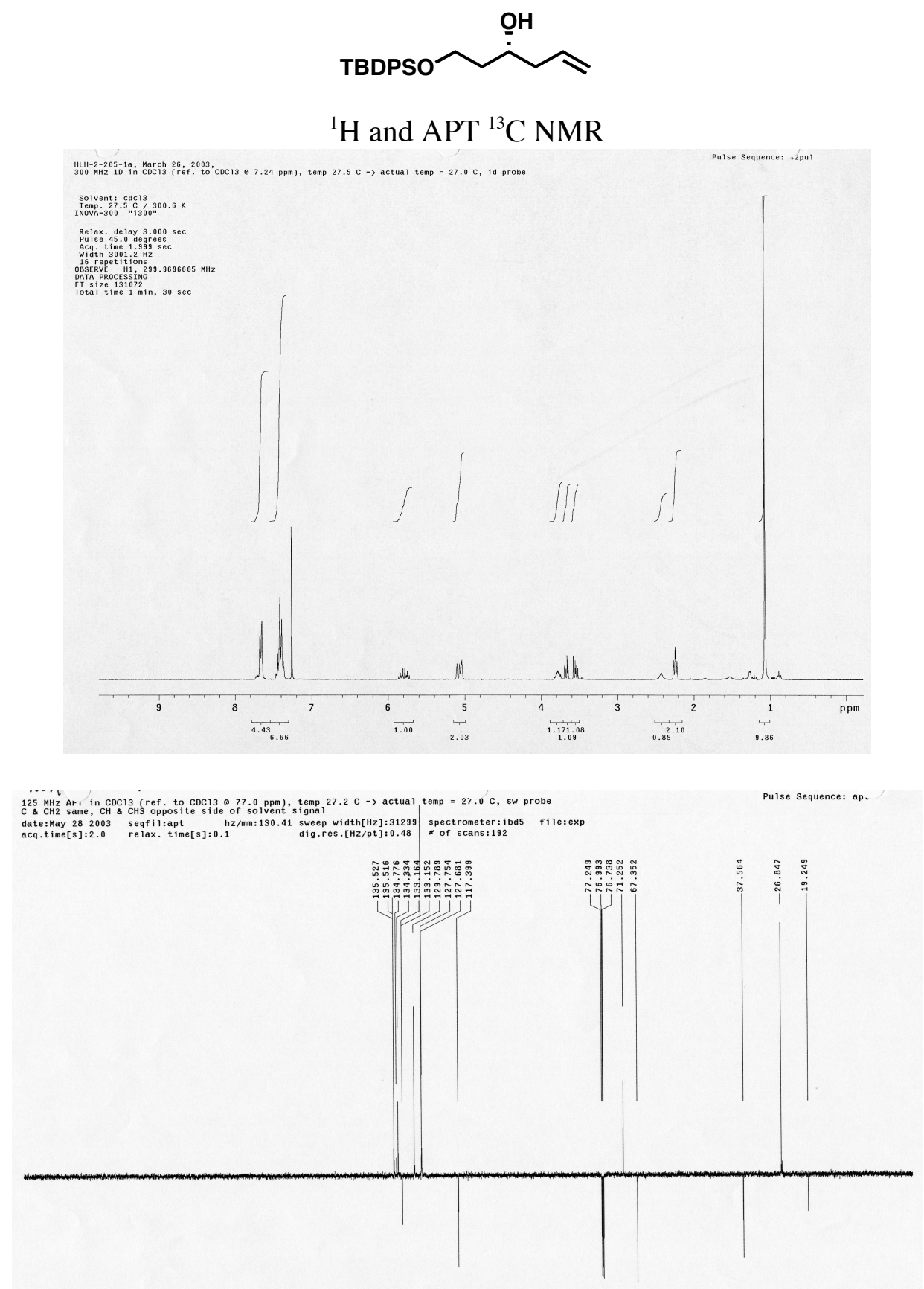

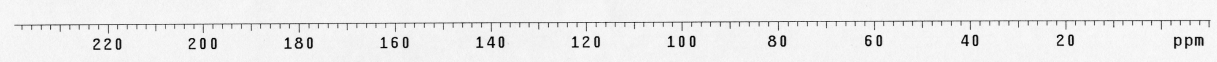


(1R)-3-Methyl-1-phenyl-3-buten-1-ol (11)<smiles>C=C(C)C[C@H](O)c1ccccc1</smiles>

\section{${ }^{1} \mathrm{H}$ and $\mathrm{APT}{ }^{13} \mathrm{C}$ NMR}
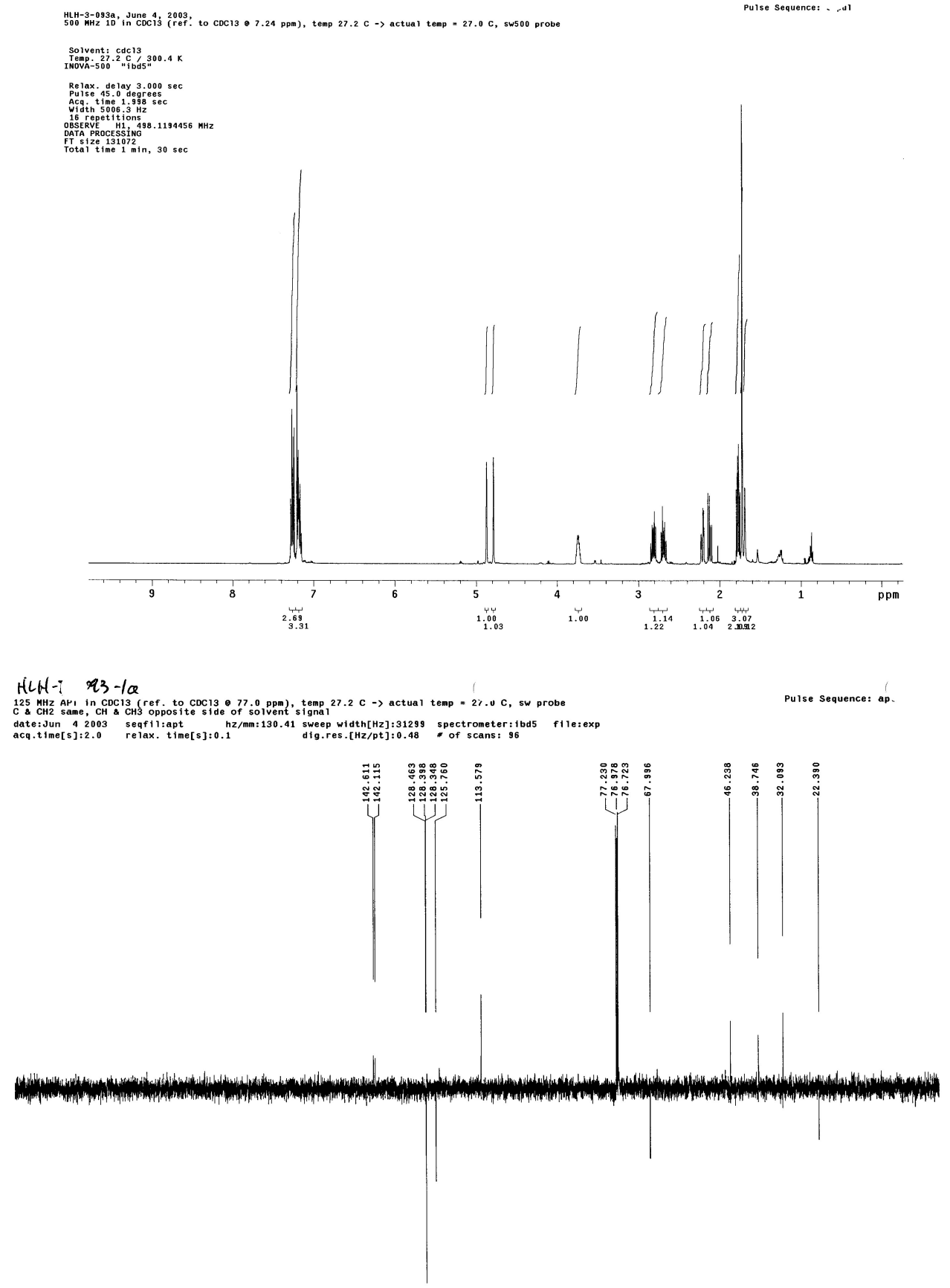

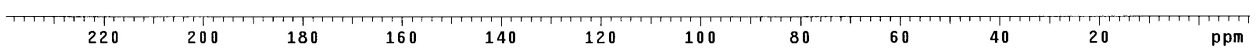


(3R)-5-Methyl-1-phenyl-5-hexen-3-ol (12)<smiles>C=C(C)C[C@H](O)CCc1ccccc1</smiles>

${ }^{1} \mathrm{H}$ and $\mathrm{APT}{ }^{13} \mathrm{C}$ NMR
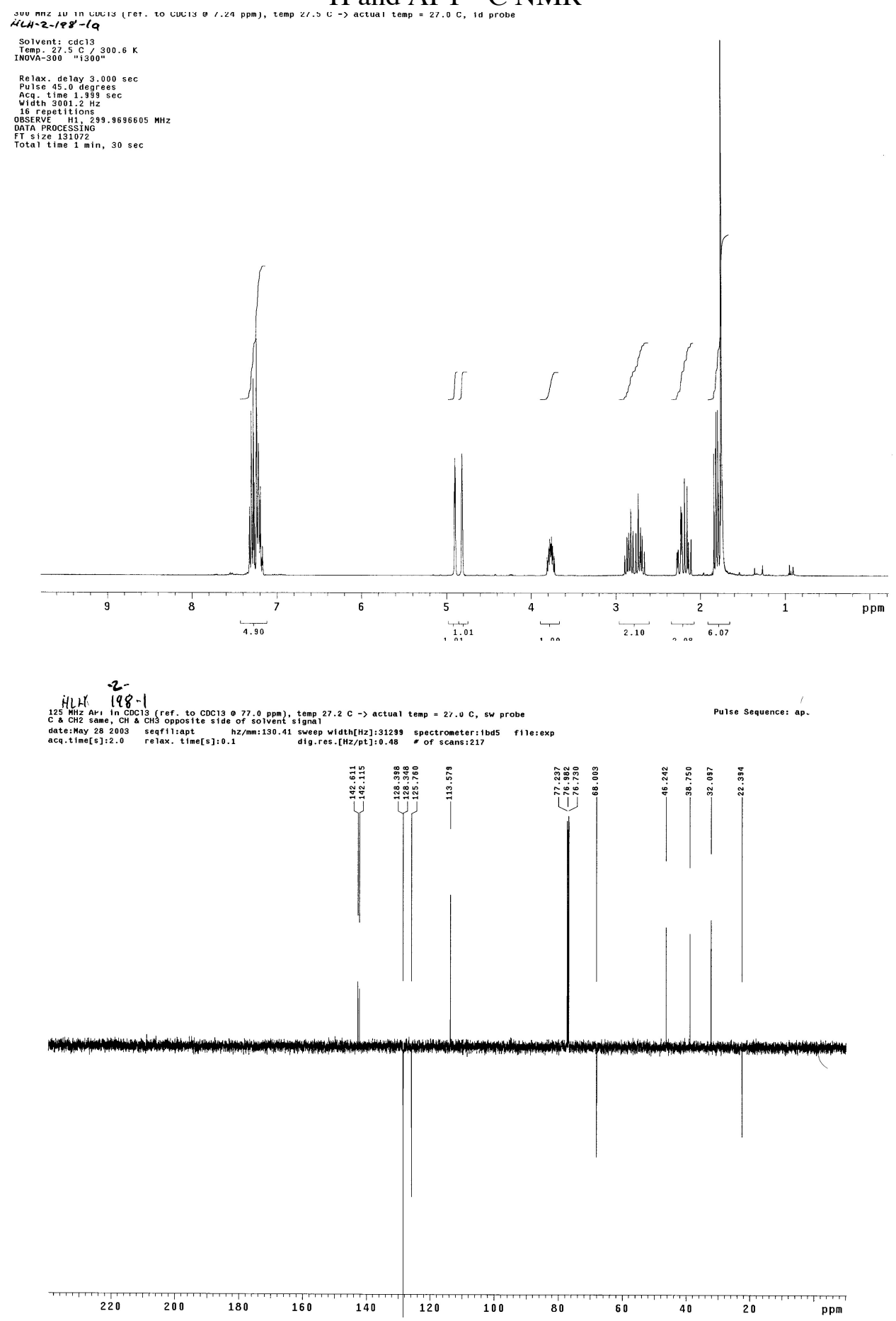
(3S)-1-( $t$-Butyldiphenylsilyloxy)-5-methyl-5-hexen-3-ol (13)
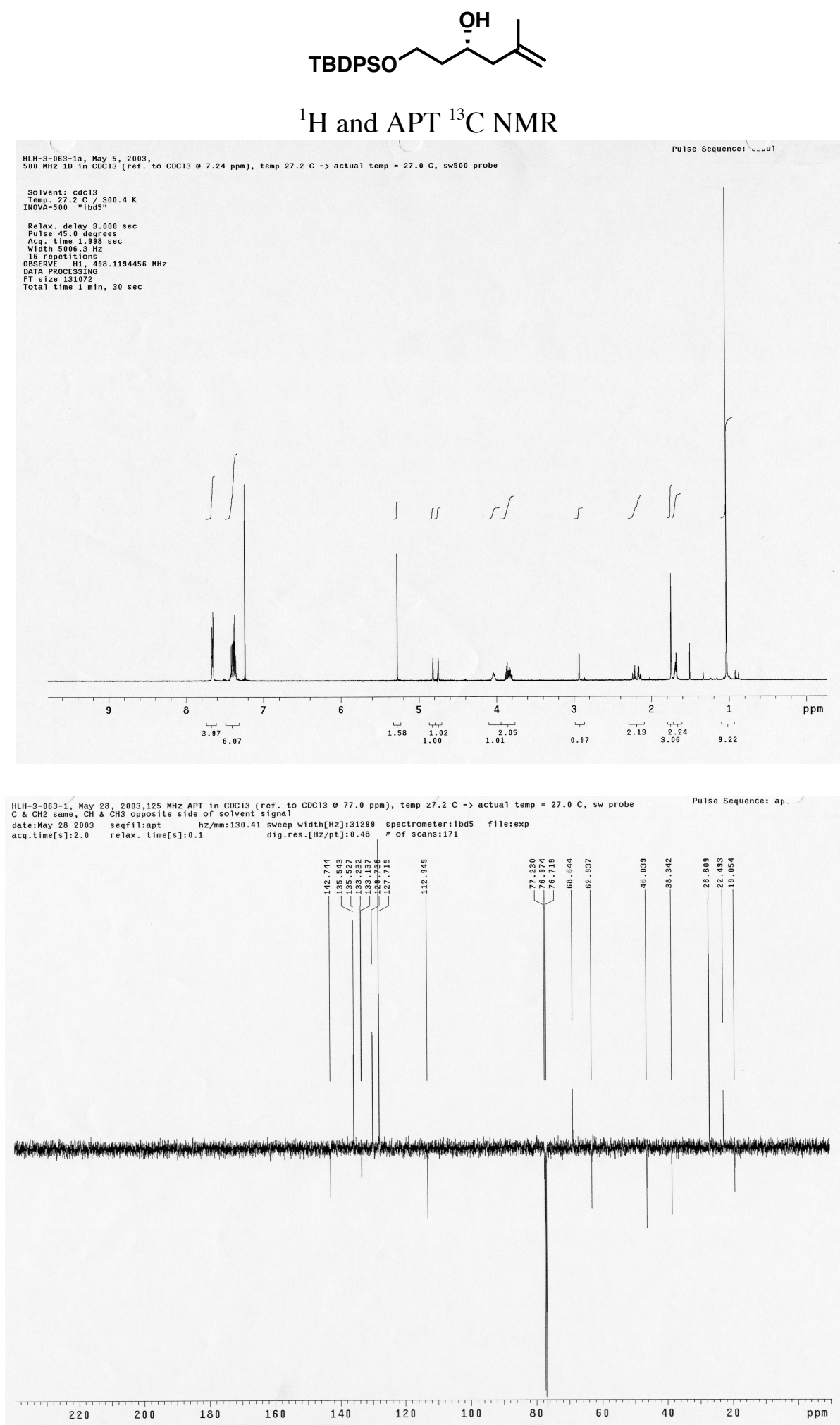
(2R)-1-(Benzyloxy)-4-methyl-4-penten-2-ol (14)

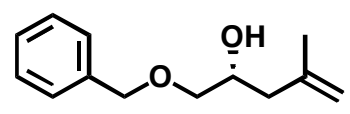

\section{${ }^{1} \mathrm{H}$ and $\mathrm{APT}{ }^{13} \mathrm{C}$ NMR}

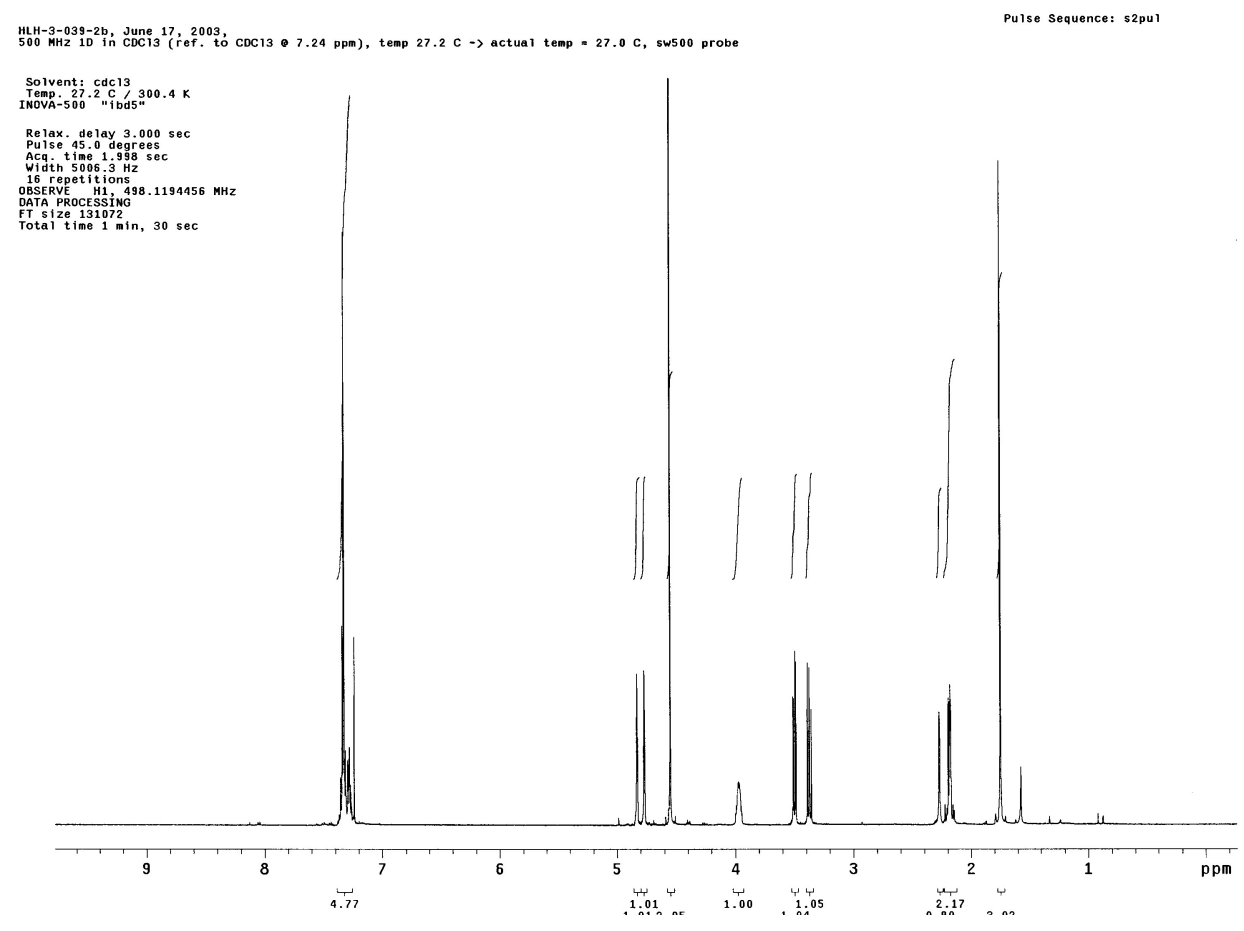

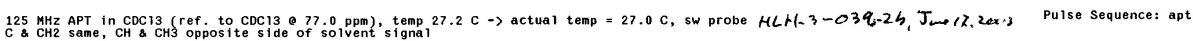

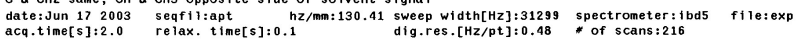

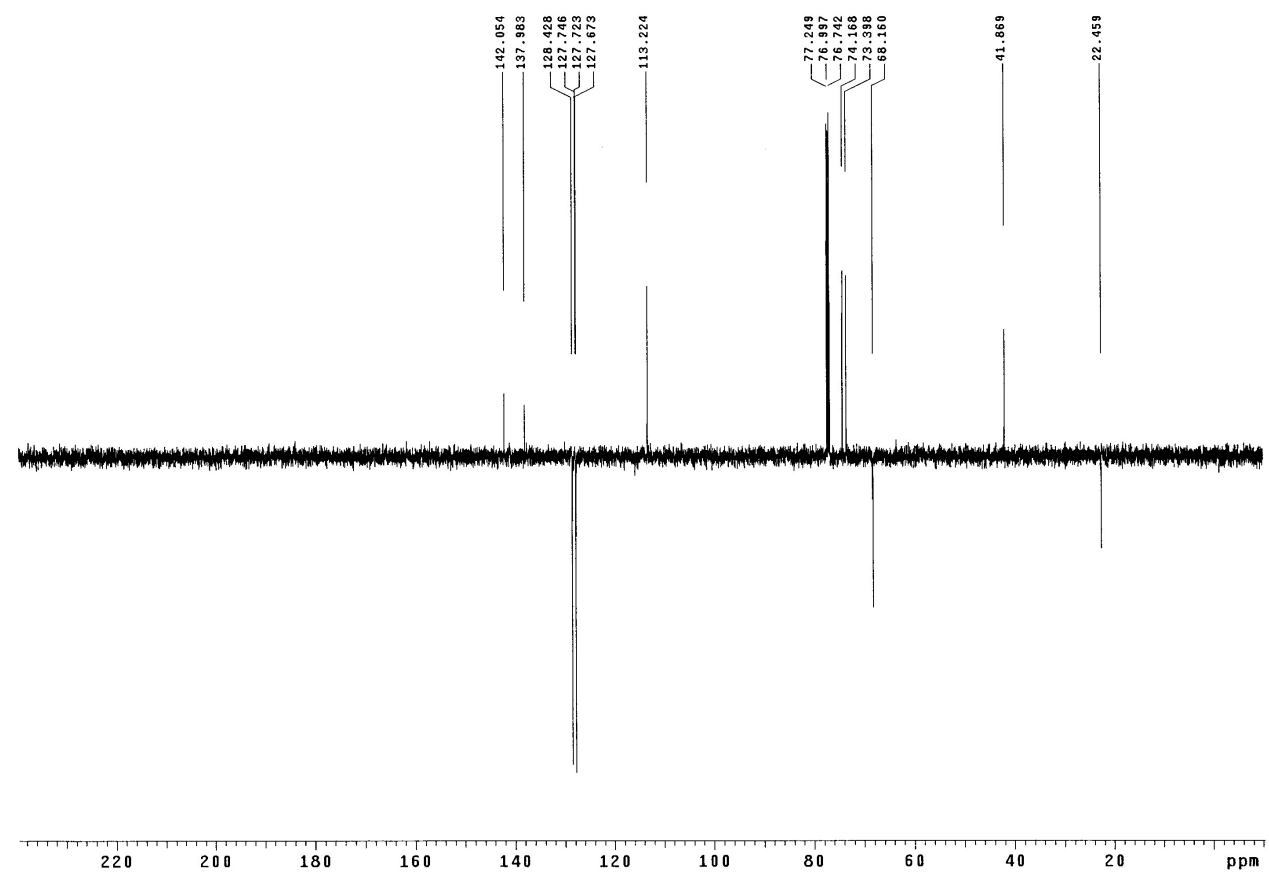


(2R)-1-( $t$-Butyldimethylsilyloxy)-4-methyl-4-penten-2-ol (15)
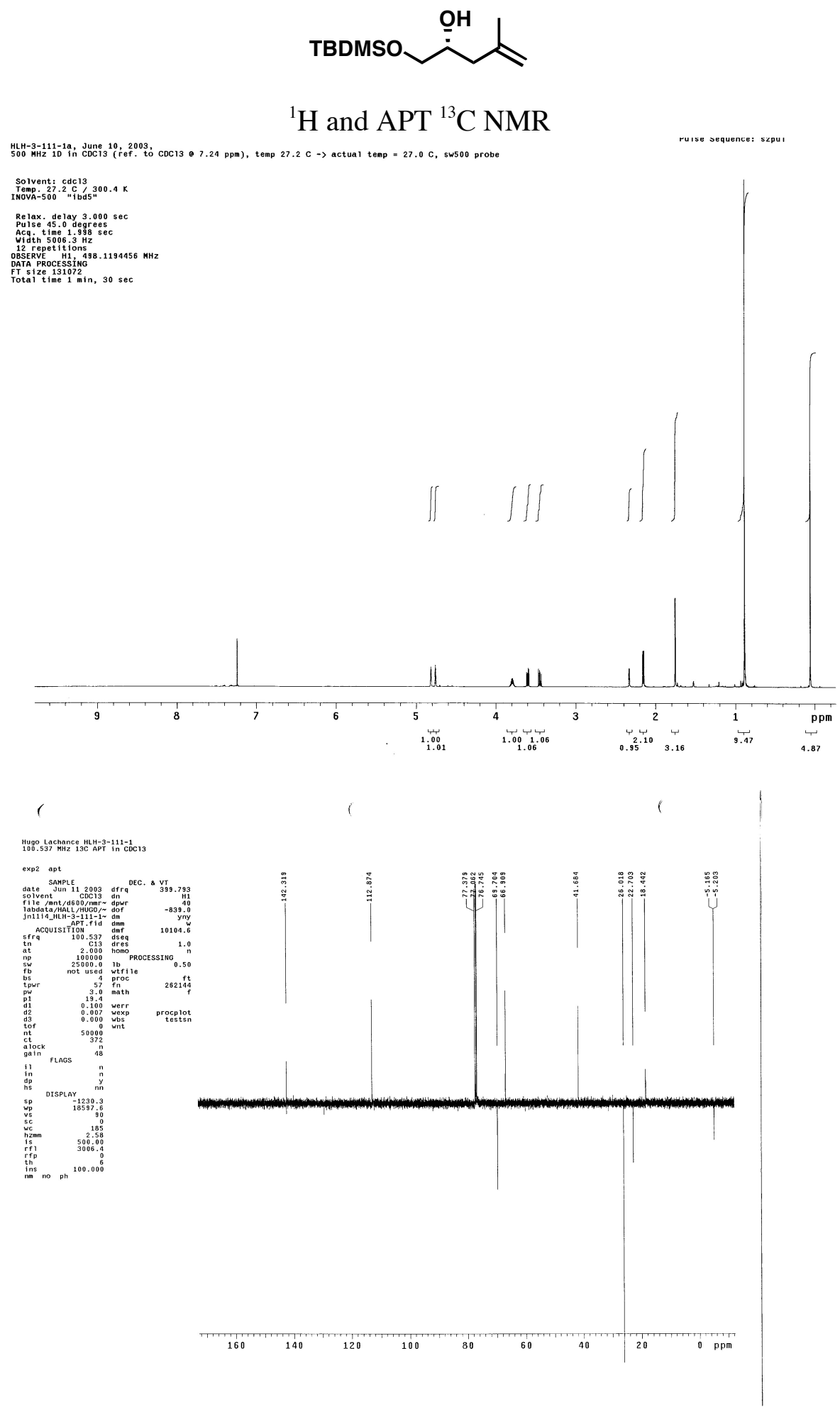
$(1 R, 2 R)$-2-Methyl-1-phenyl-3-buten-1-ol (16)

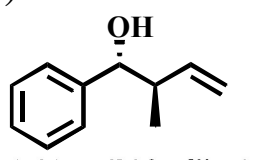

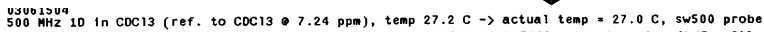

sweep width[Hz]:5006 spectrometer: ibd5 file:/mnt/d600/hall nmrdata/Xfaosong/al ly lboration/03061504.fid

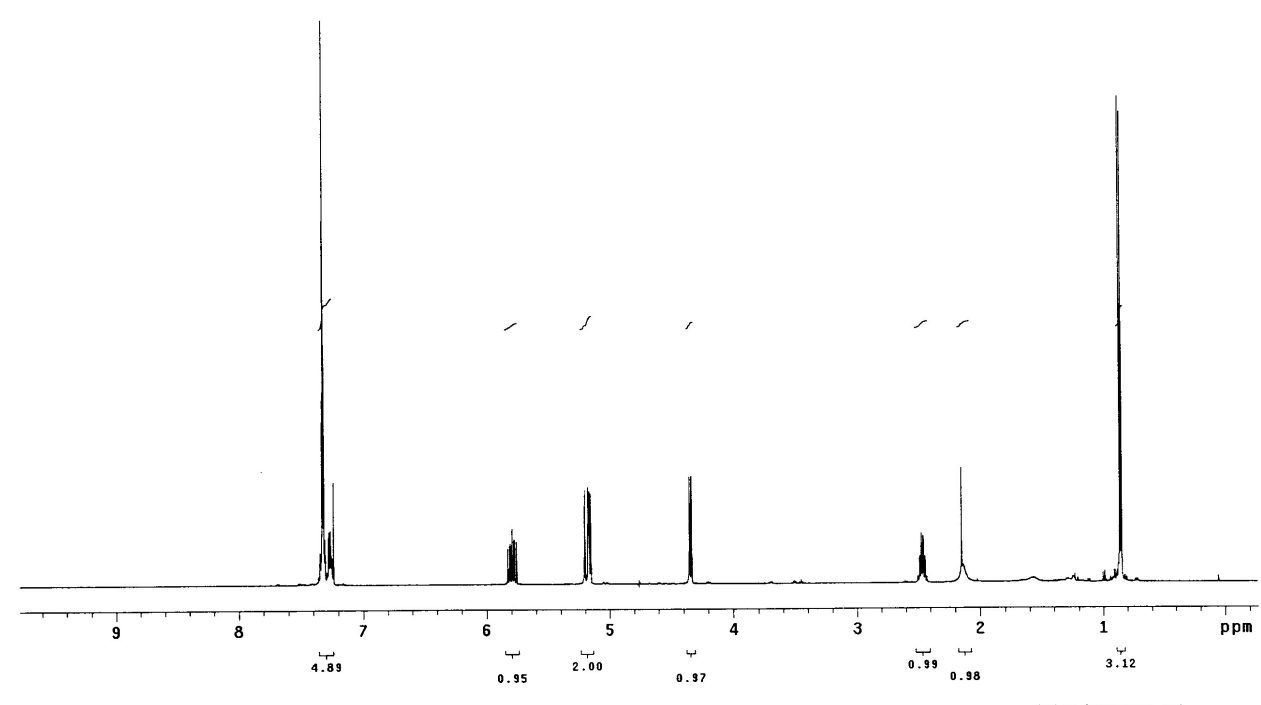

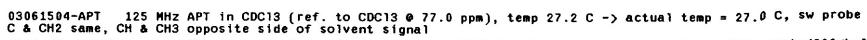

Pulse Sequence: apt

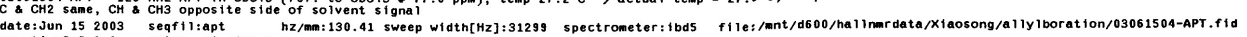

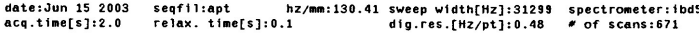
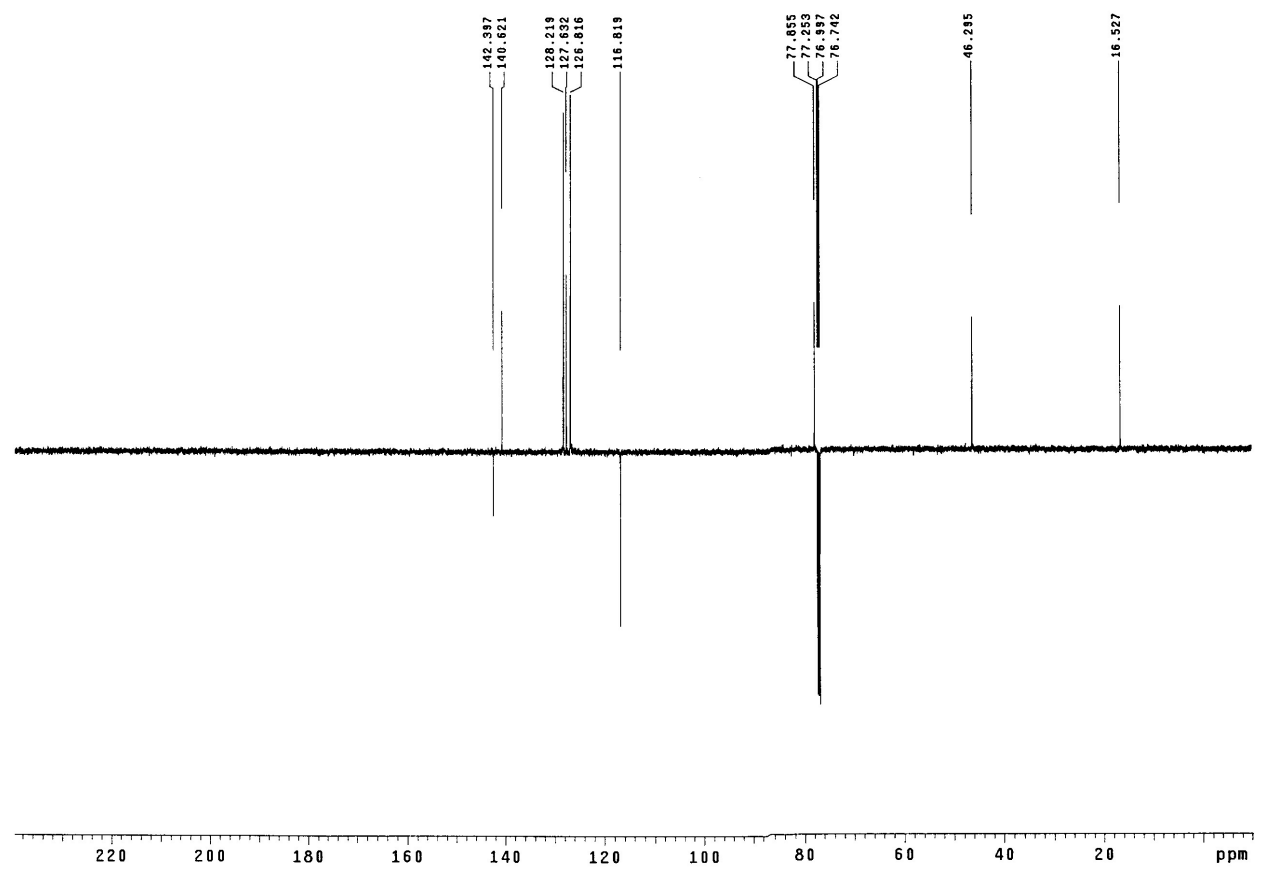
(3S, 4R)-4-Methyl-1-phenyl-5-hexen-3-ol (17)

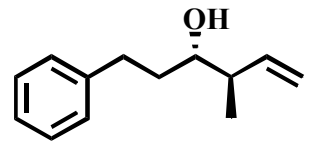

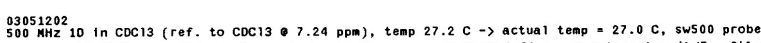

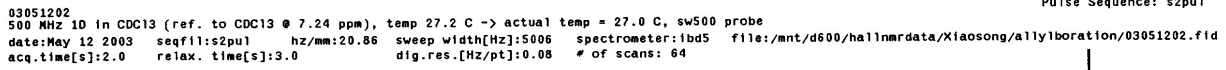

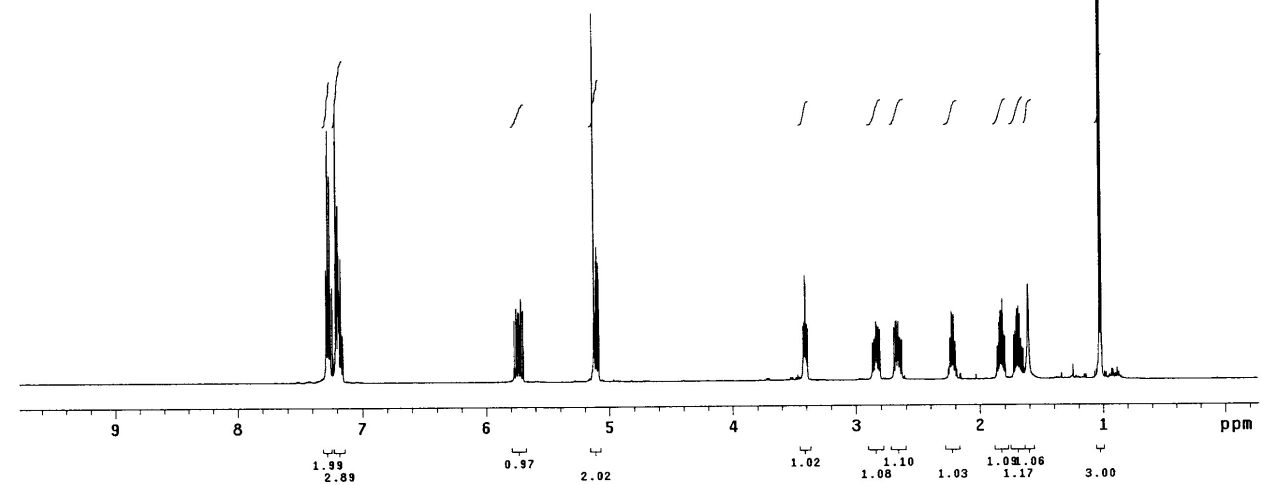

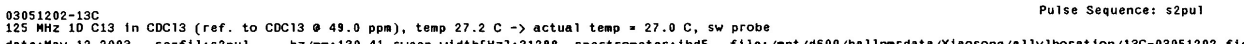

Pulse Sequence: 52pur

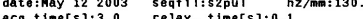

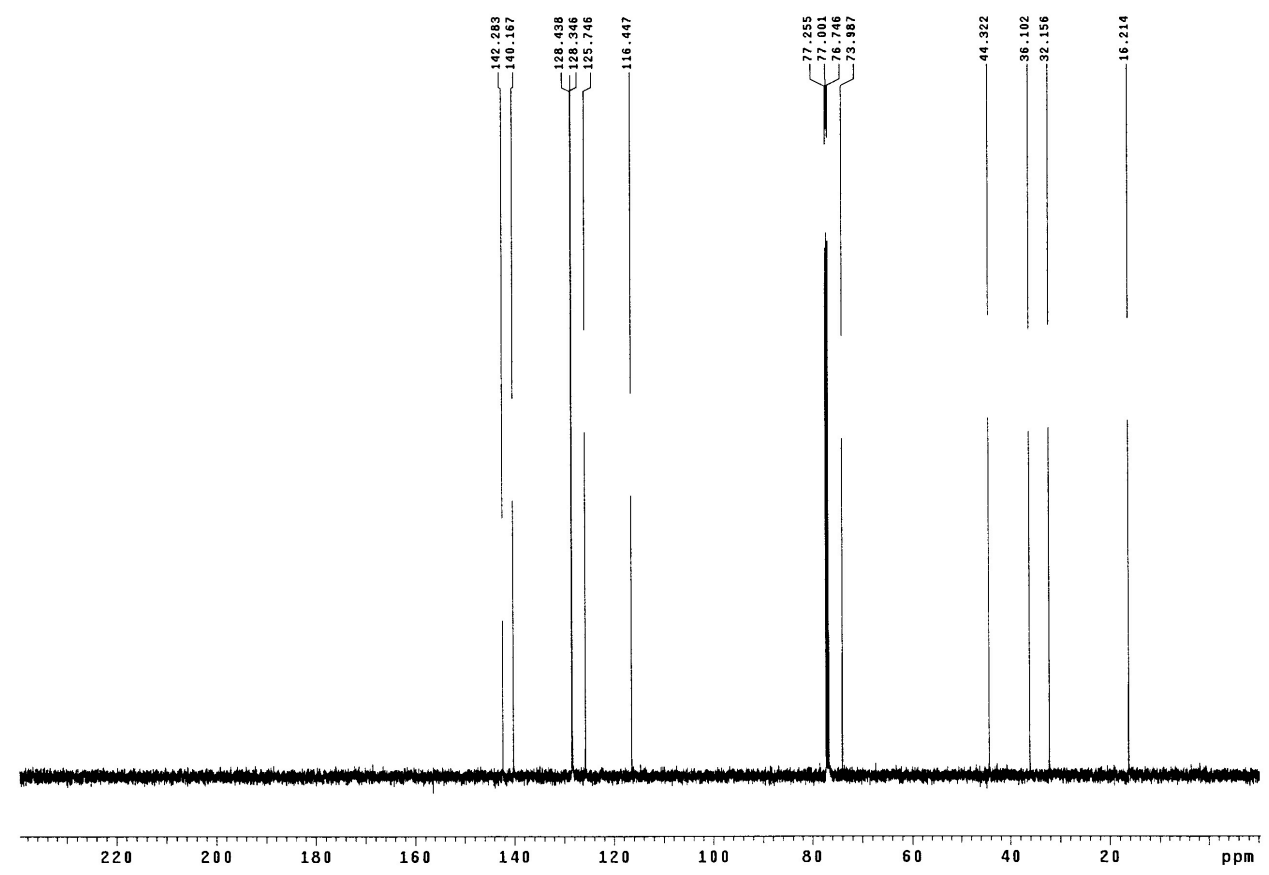


(3S, 4R)-1-( $t$-Butyldiphenylsilyloxy)-4-methyl-5-hexen-3-ol (18)

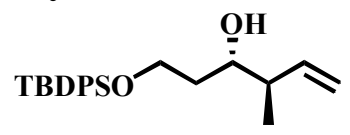

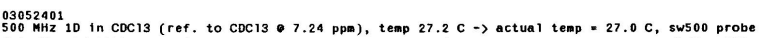

Pulse Sequence: s2pur

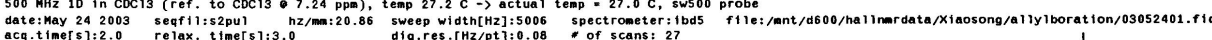
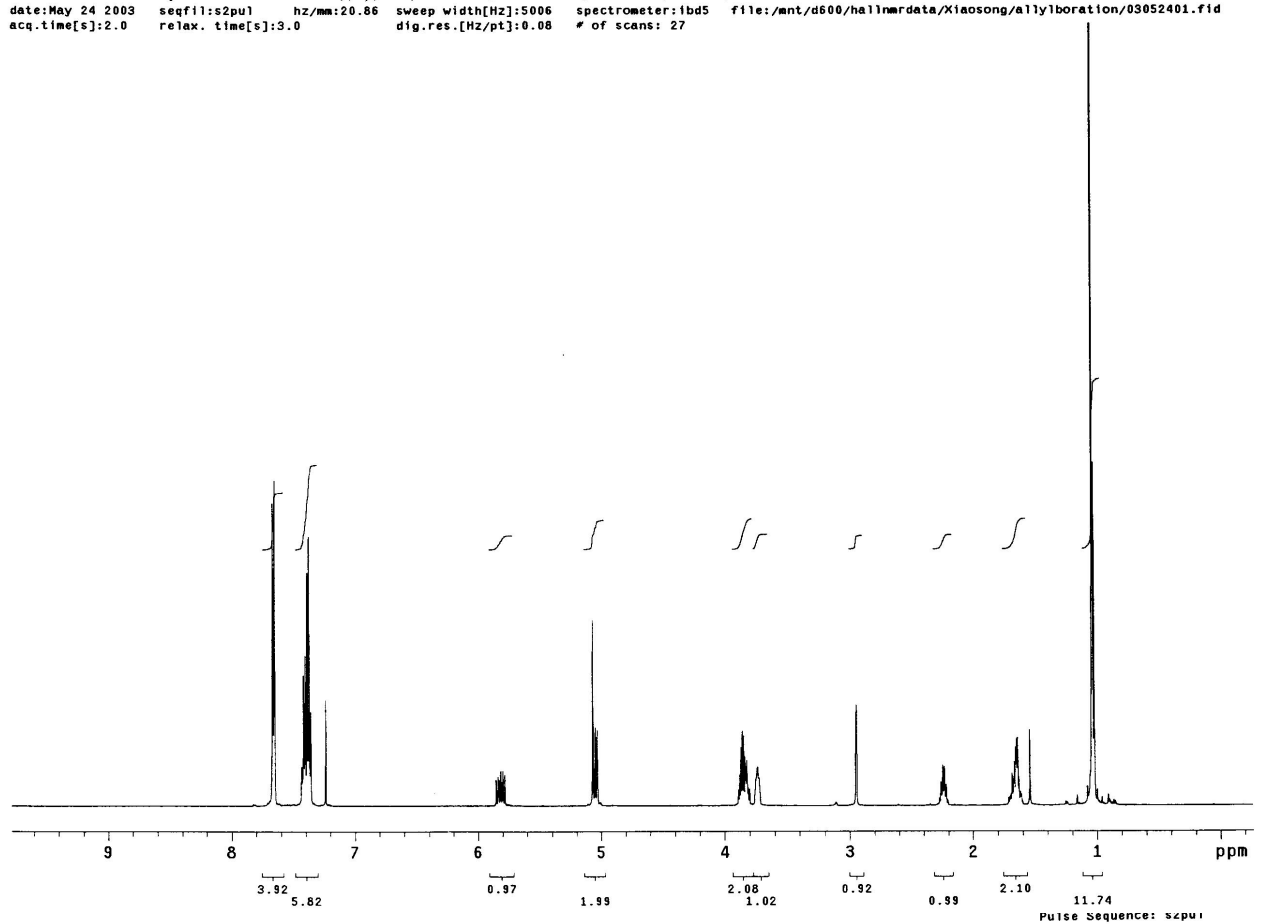

$03052401-13 \mathrm{C}$
125
$\mathrm{HHz}$
10

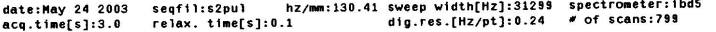

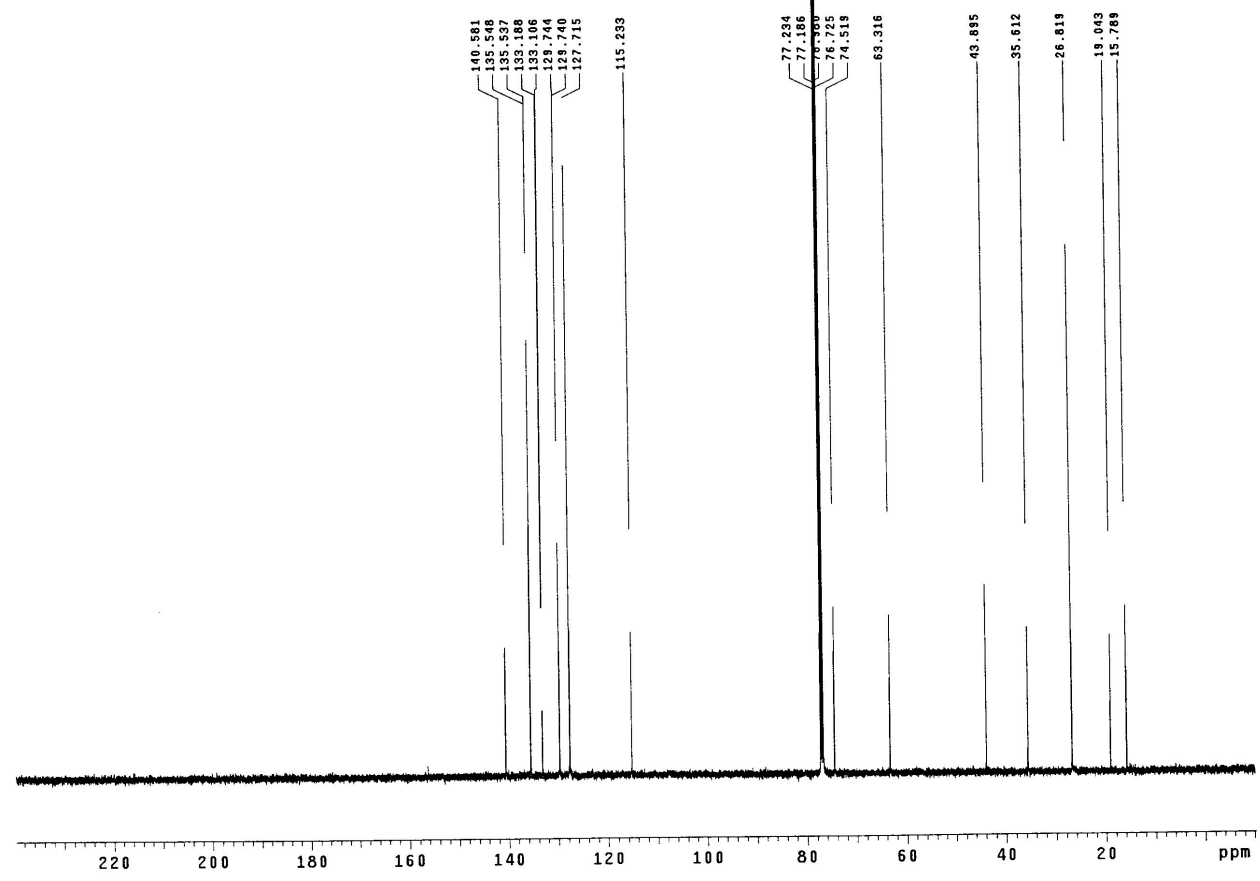


(2R, 3R)-1-( $t$-Butyldimethylsilyloxy)-3-methyl-4-penten-2-ol (19)

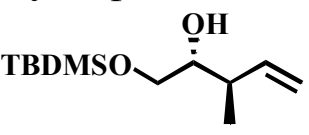

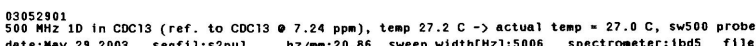

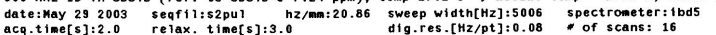

Pulse Sequence: s2pur

res. $[\mathrm{Hz} / \mathrm{pt}]: 0.08$ of scans: 16 G

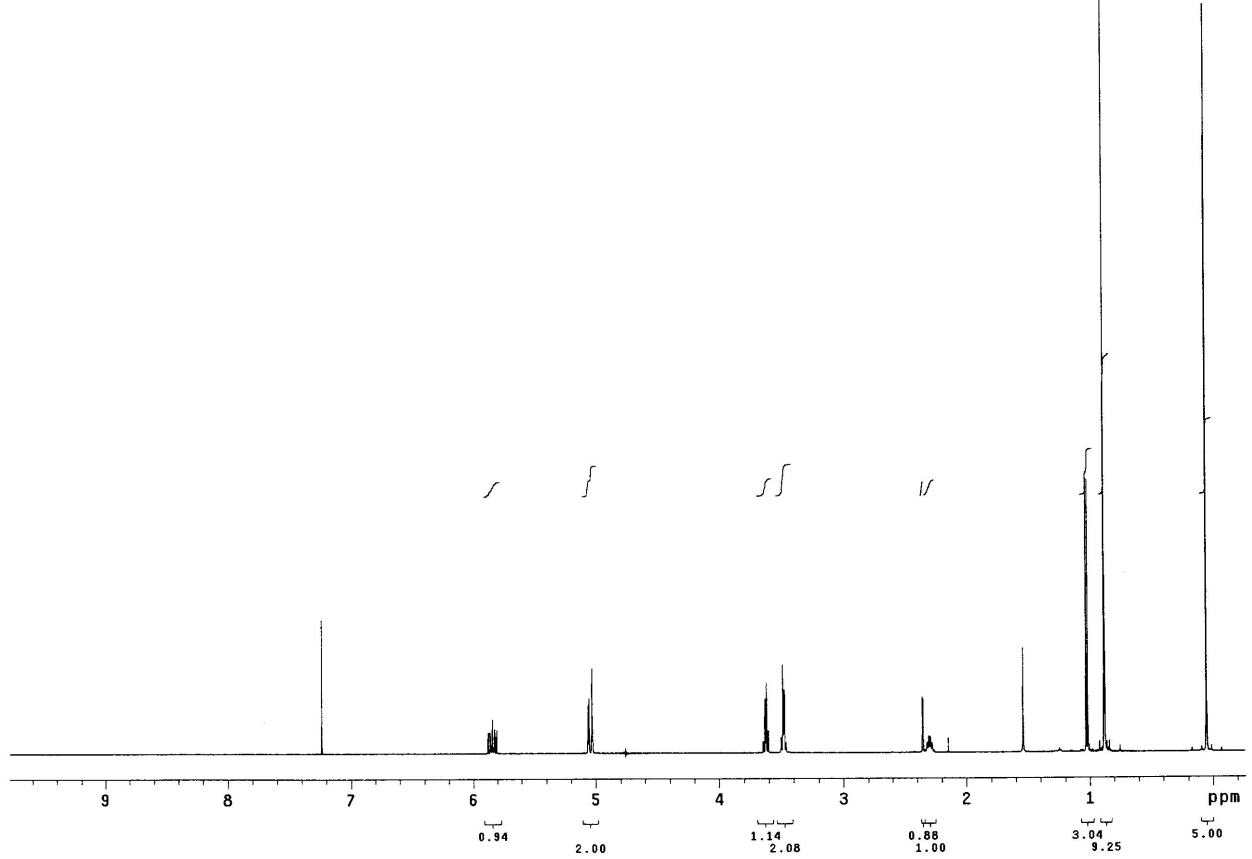

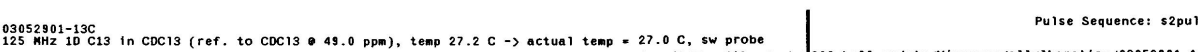

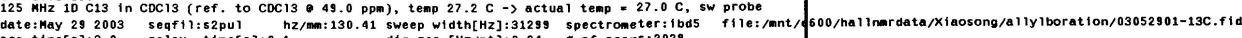

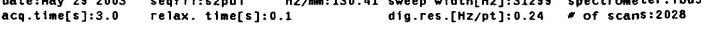
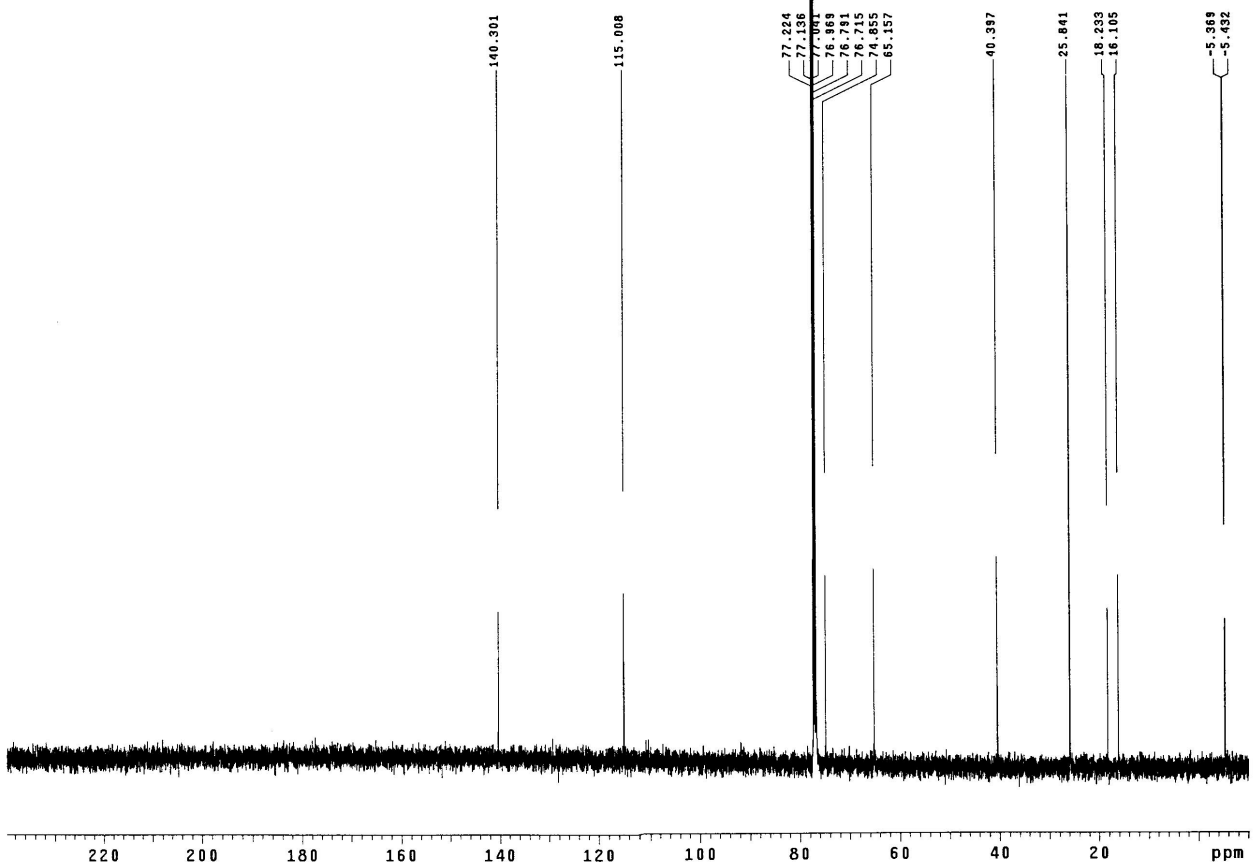

80

60 
$(1 R, 2 S)$-2-Methyl-1-phenyl-3-buten-1-ol (20)<smiles>C=C[C@H](C)[C@H](O)c1ccccc1</smiles>

\section{${ }^{1} \mathrm{H}$ and $\mathrm{APT}{ }^{13} \mathrm{C}$ NMR}
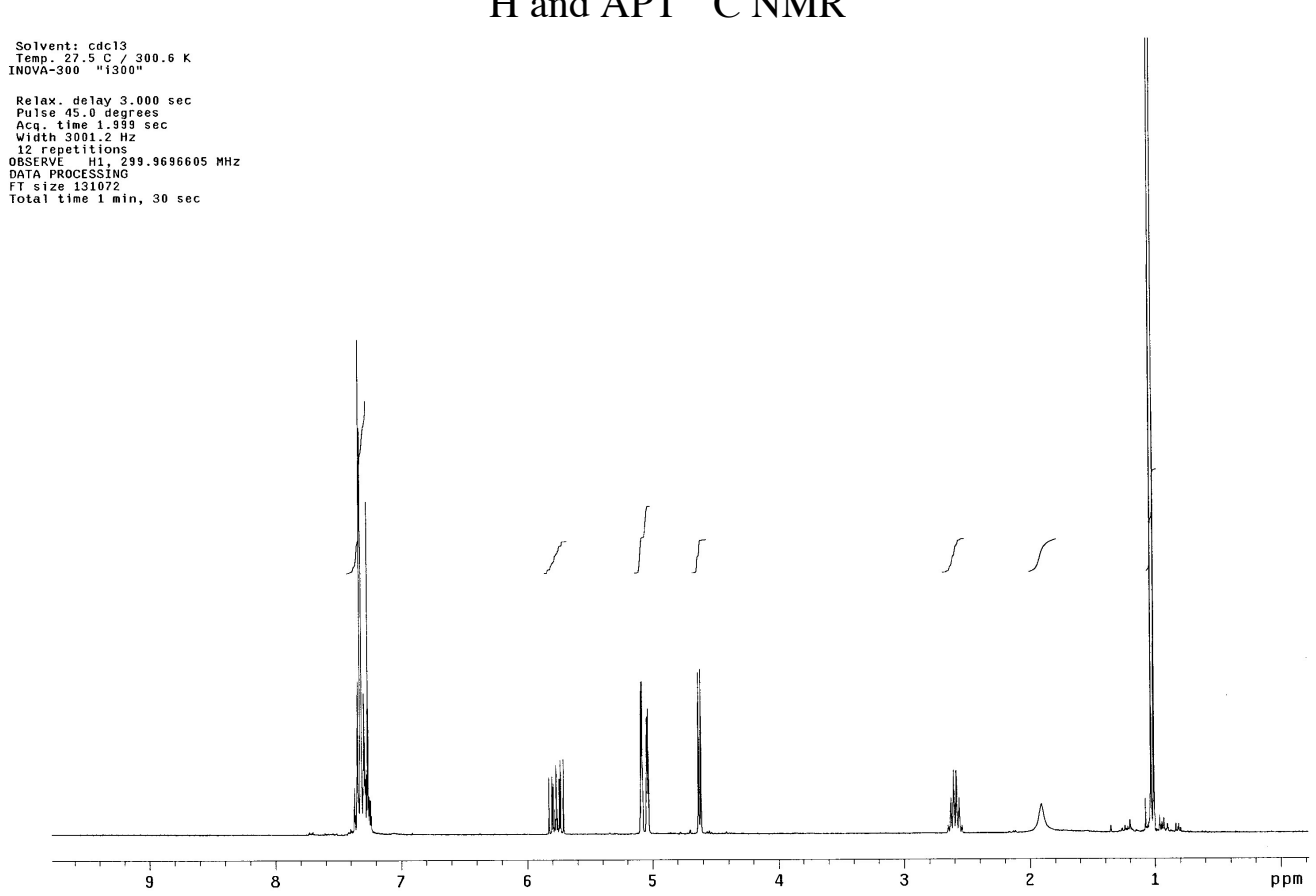

MGH-14-26B
125 MHZ APT in CDC13 (ref. to $C D C 13$ o 77.0 ppm), temp $27.2 \mathrm{C}->$ actual temp $=27.0 \mathrm{C}$, sw probe
C \& CH2 same, CH \& CH3 opposite side of solvent'signal

Pulse Sequence: ap

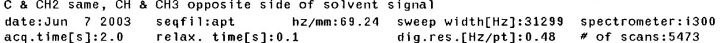

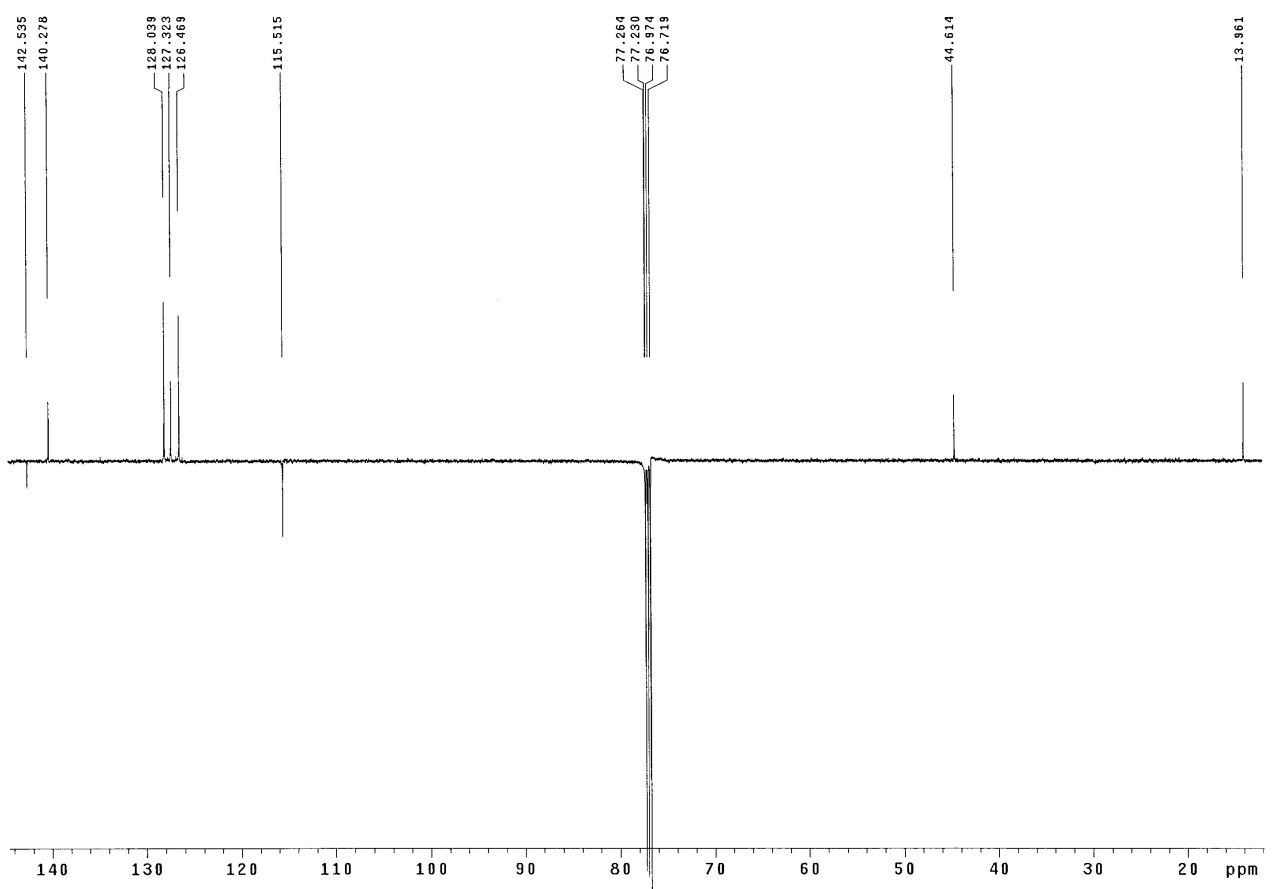


(3S, 4S)-4-Methyl-1-phenyl-5-hexen-3-ol (21)

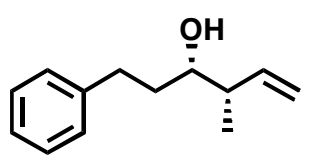

\section{${ }^{1} \mathrm{H}$ and APT ${ }^{13} \mathrm{C}$ NMR}

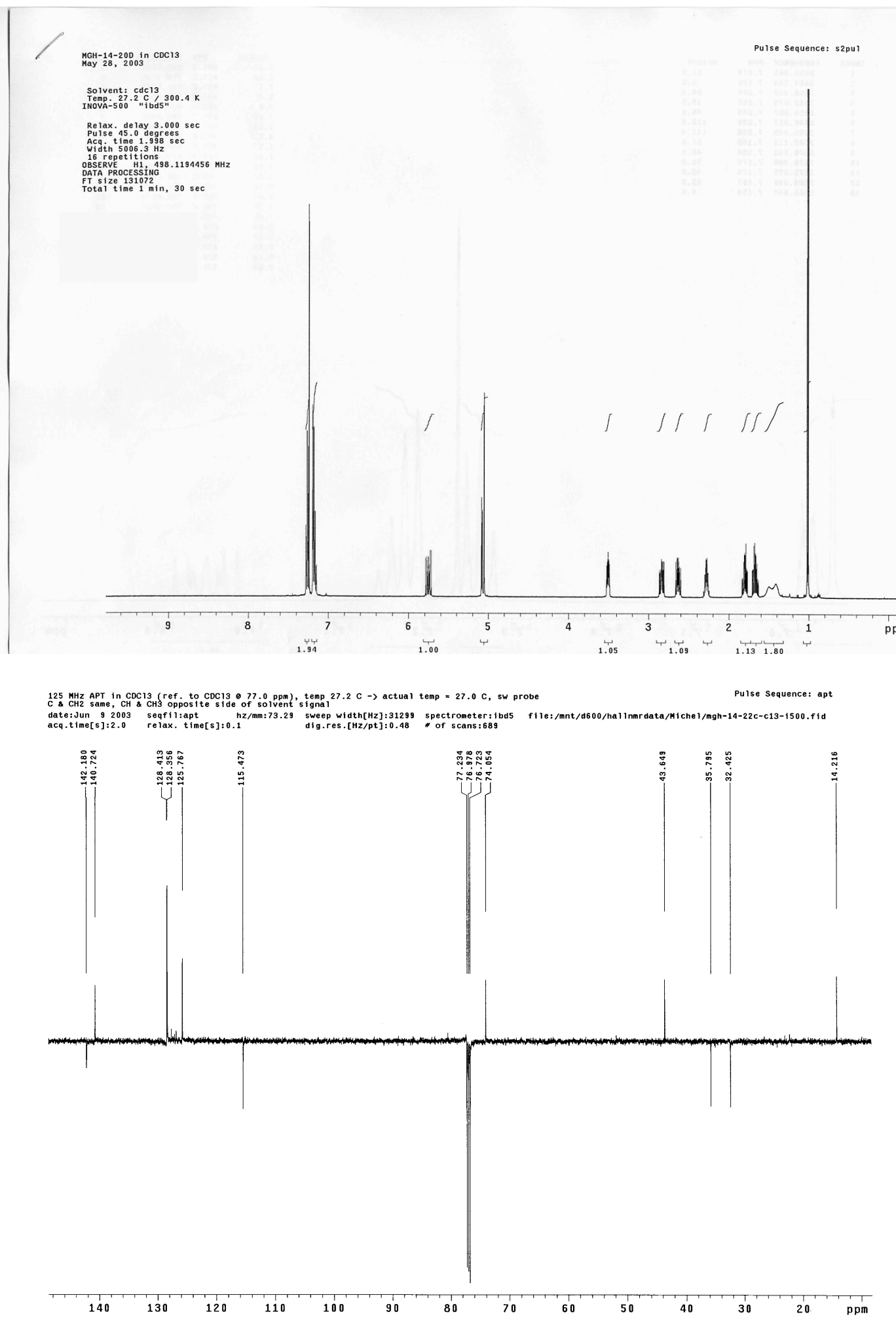


$(3 S, 4 S)-1-(t$-Butyldiphenylsilyloxy)-4-methyl-5-hexen-3-ol (22)

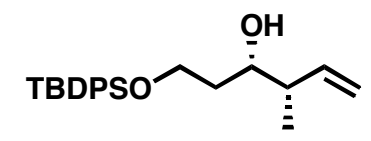

${ }^{1} \mathrm{H}$ and $\mathrm{APT}{ }^{13} \mathrm{C}$ NMR

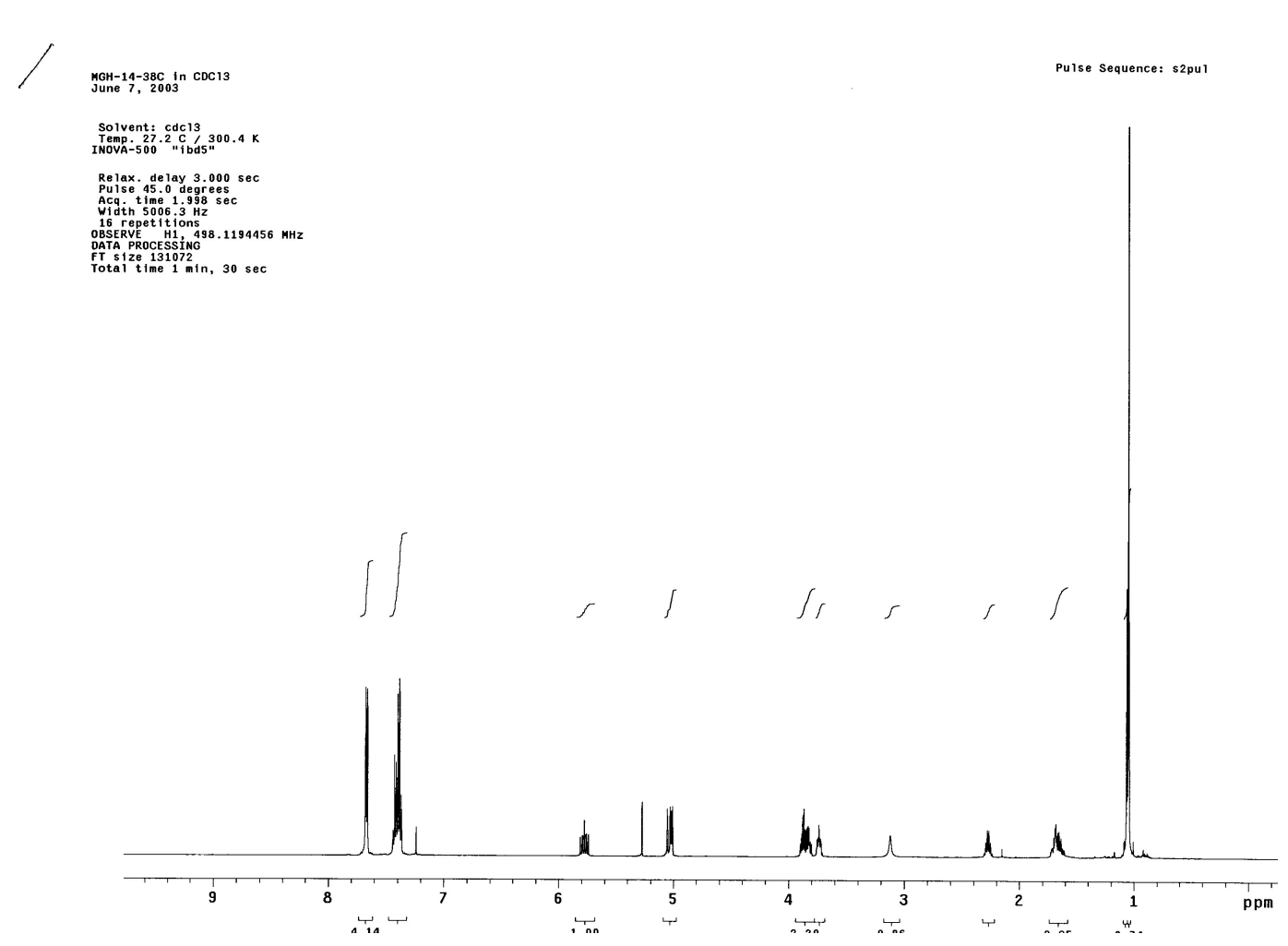

$\mathrm{MGH}-14-38 \mathrm{C}$ in $\mathrm{CDC} 13$
June 7,2003

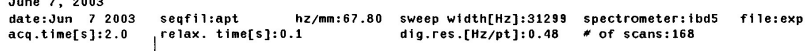
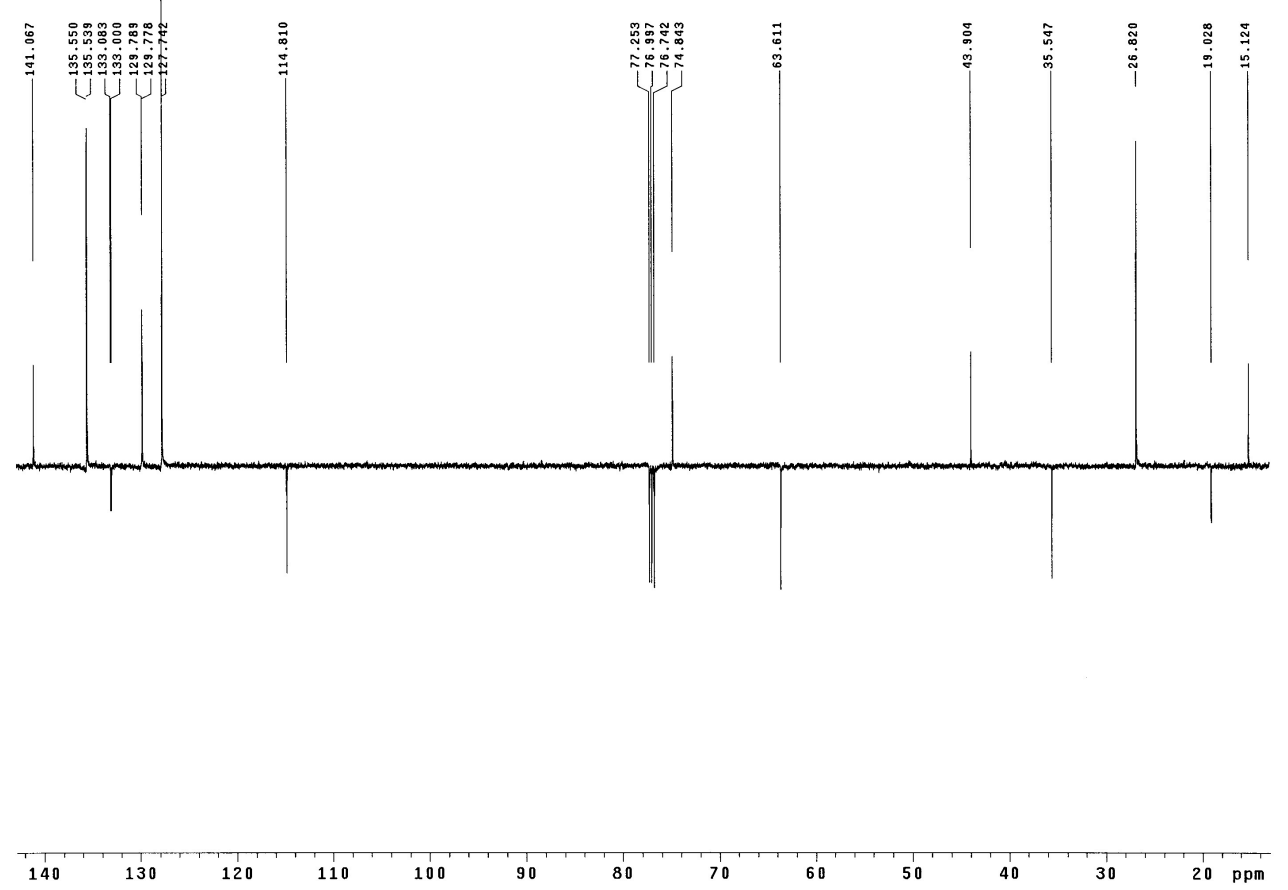
$(2 R, 3 S)-1$-( $t$-Butyldimethylsilyloxy)-3-methyl-4-penten-2-ol (23)

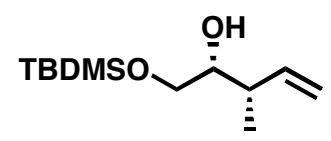

${ }^{1} \mathrm{H}$ and $\mathrm{APT}{ }^{13} \mathrm{C}$ NMR

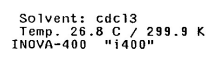

Relax. delay $3.000 \mathrm{sec}$
Pulse 45.0 degrees

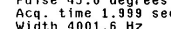

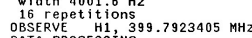

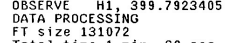

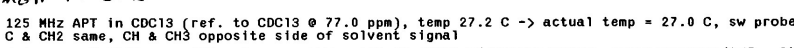

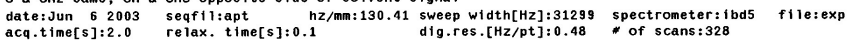

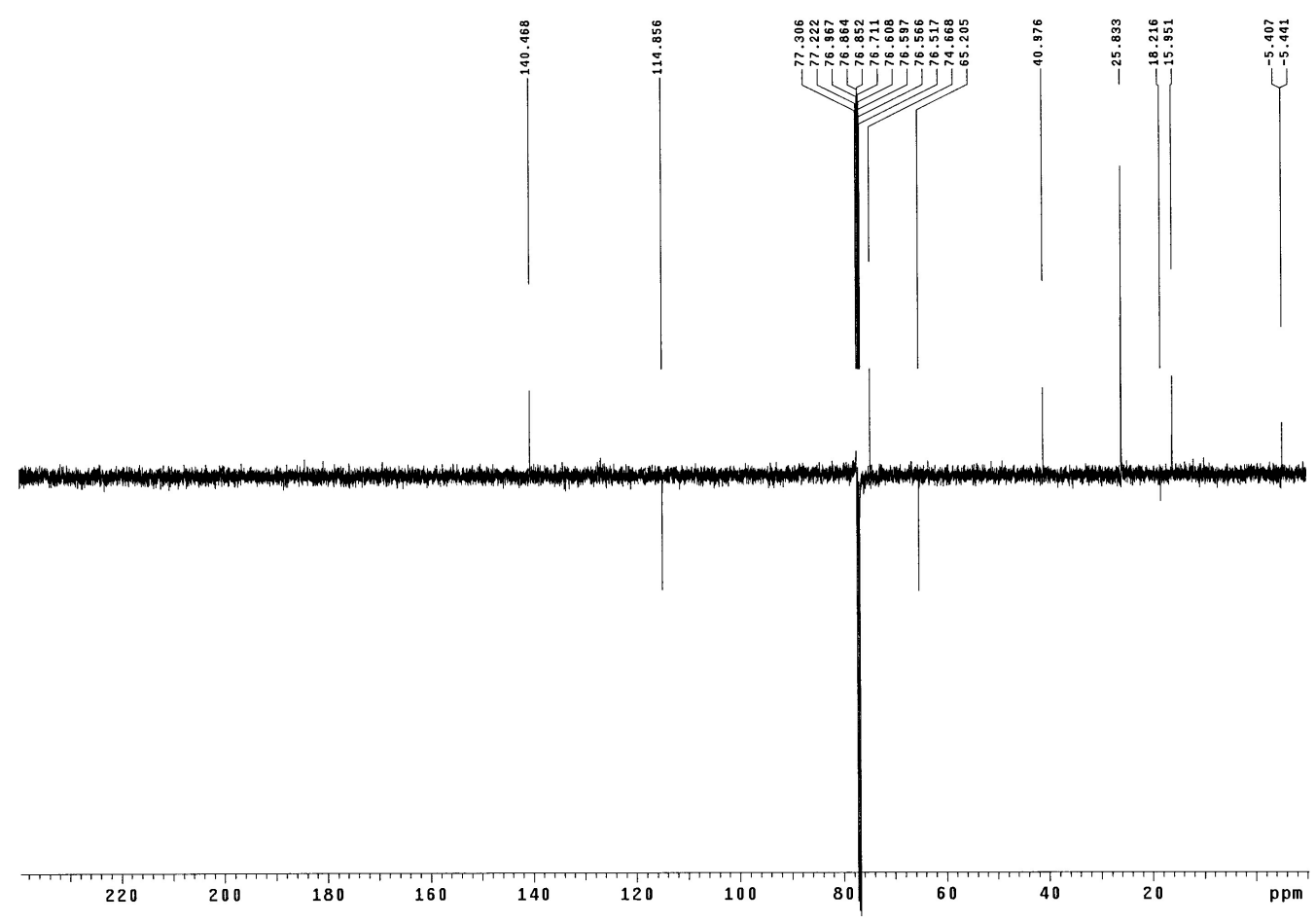




\section{Representative HPLC chromatograms}

Typical Chiral HPLC for reactions with boronate 1 a. Compound 6

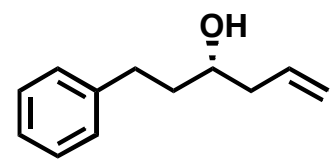

Racemic sample

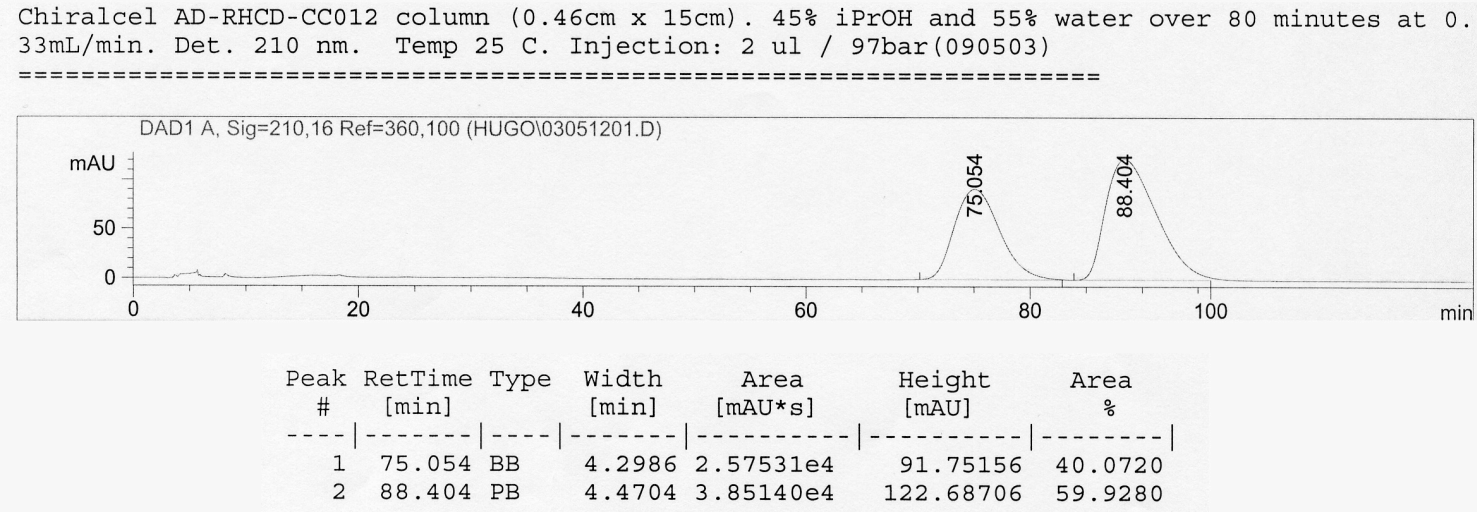

Enantioselective reaction

Chiralcel AD-RHCD-CC012 column $(0.46 \mathrm{~cm} \times 15 \mathrm{~cm})$. 45\% iPrOH and 55\% water over 80 minutes at 0. $33 \mathrm{~mL} / \mathrm{min}$. Det. $210 \mathrm{~nm}$. Temp 25 C. Injection: 2 ul / 97bar(090503)
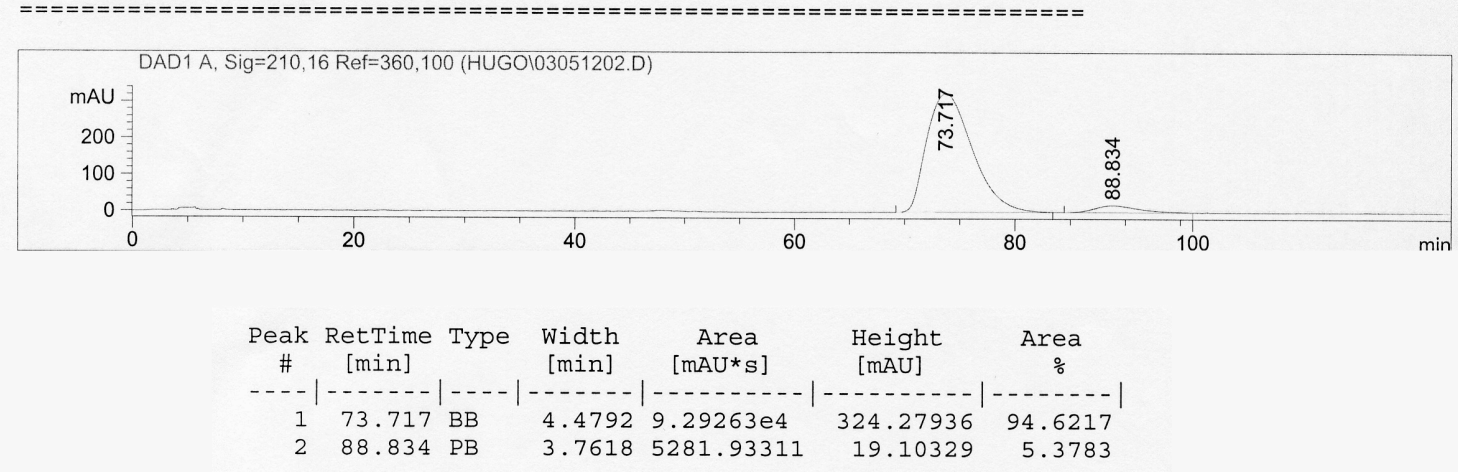
Typical Chiral HPLC for reactions with boronate 2a.

Compound 12

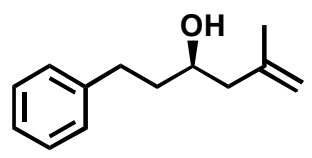

Racemic sample

Chiralcel OD-RHCD-CCO12 column $(0.46 \mathrm{~cm} \mathrm{x} \mathrm{15cm).} 45 \%$ iPrOH and 55\% water over 60 minutes at $0.4 \mathrm{~mL} / \mathrm{min}$. Det. $210 \mathrm{~nm}$. Temp $25 \mathrm{C}$

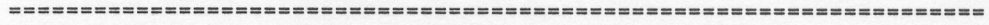

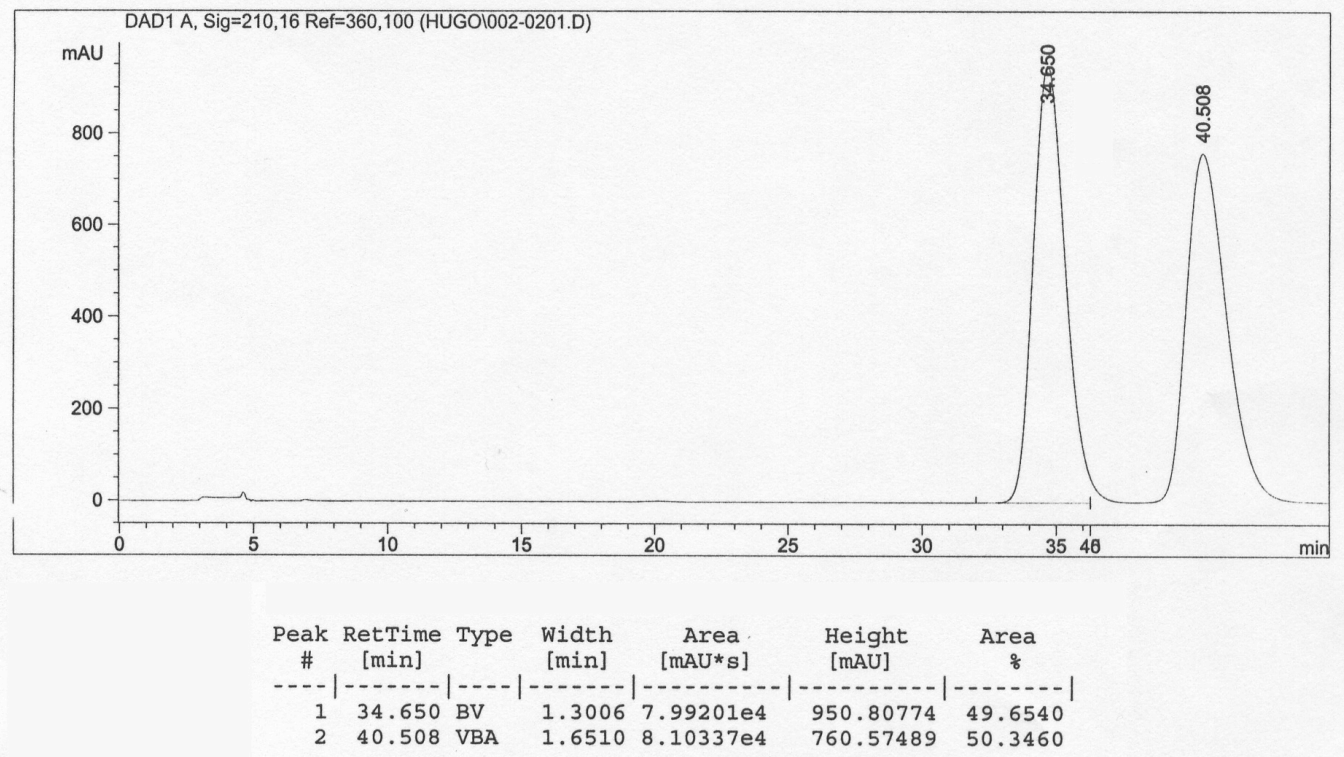

Enantioselective reaction

Chiralcel ${ }^{-} \mathrm{OD}-\mathrm{RHCD}-\mathrm{CCO} 12$ column $\left(0.46 \mathrm{~cm}^{-} \times 1 \overline{\mathrm{cm}}\right) .45 \%$ iPrOH and $55 \%$ water over 60 minutes at $0.4 \mathrm{~mL} / \mathrm{min}$. Det. $210 \mathrm{~nm}$. Temp $25 \mathrm{C}$

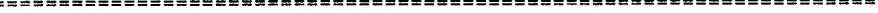

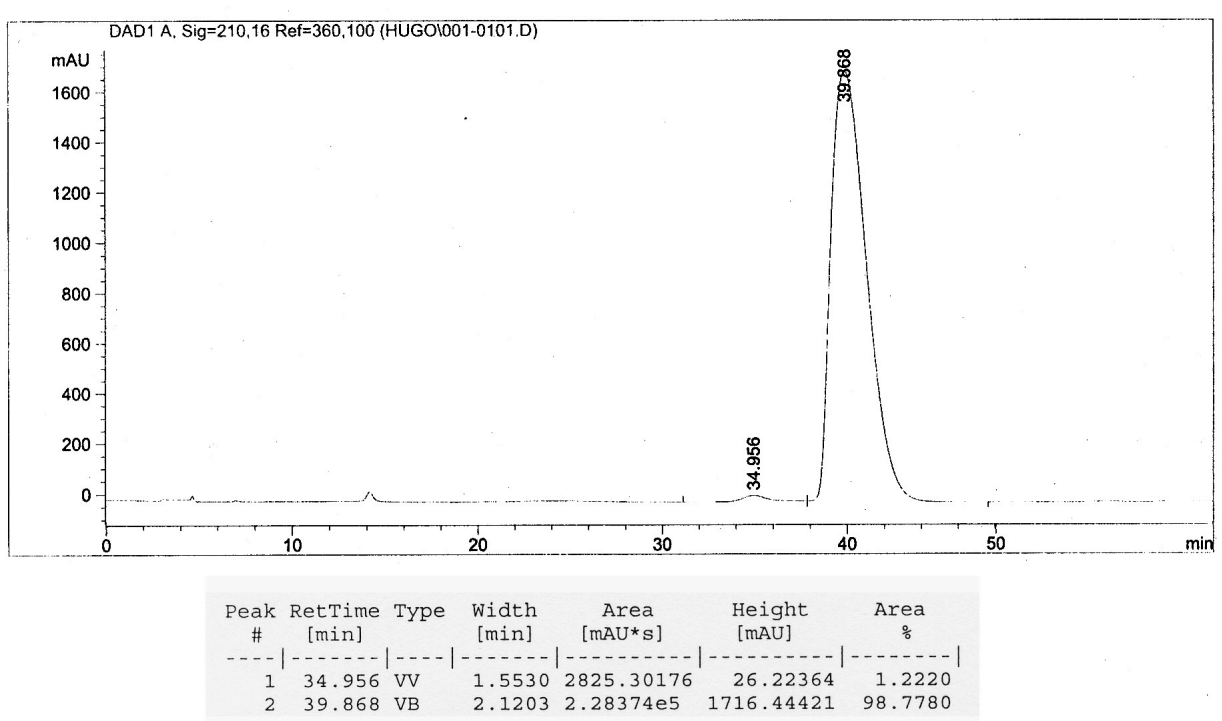


Typical Chiral HPLC for reactions with boronate 3a.

Compound 17

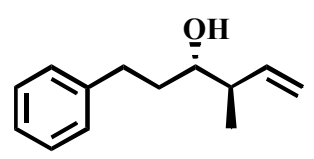

Racemic sample

chiral AD-RH HPLC with 40 isopropanol/H20 for 80 minutes; DAD detector; flow rate of 0.30 $\mathrm{ml} / \mathrm{min}$
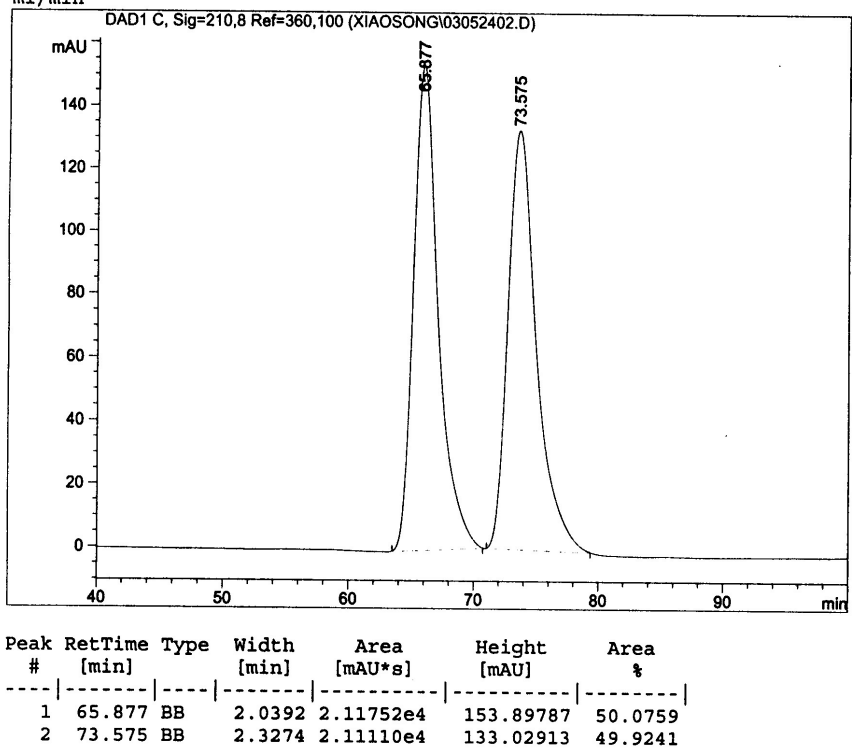

Enantioselective reaction

chiral AD-RH HPLC with 408 isopropanol/H20 for 80 minutes; DAD detector; flow rate of 0.300 $\mathrm{ml} / \mathrm{min}$

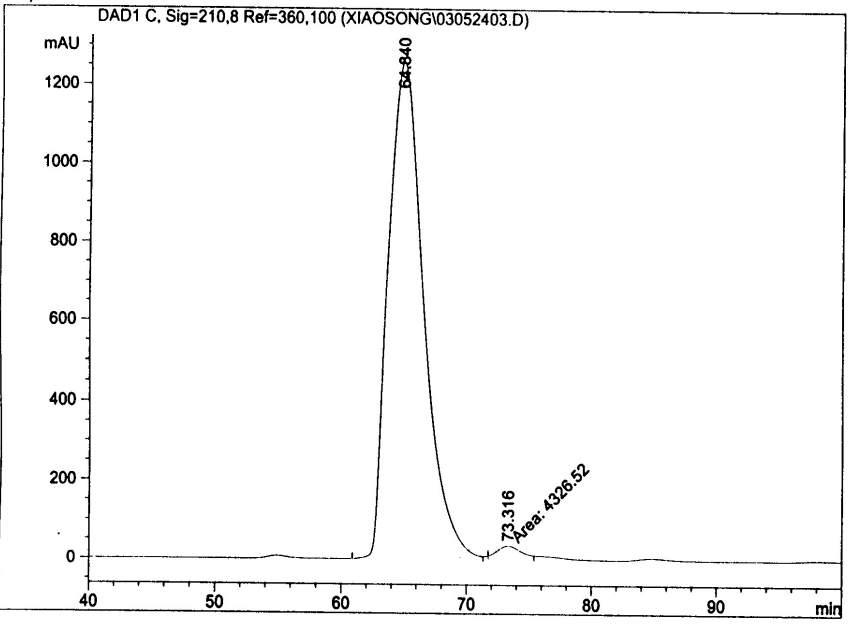

Peak RetTime Type Width Area Height Area

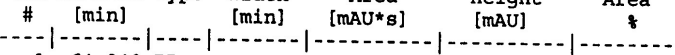

$\begin{array}{lllllll}1 & 64.840 & \mathrm{BB} & 2.7141 & 2.46126 \mathrm{e} 5 & 1256.07239 & 98.2725\end{array}$

$\begin{array}{lrlrrr}73.316 \mathrm{MM} & 2.2231 & 4326.52490 & 32.43549 & 1.7275\end{array}$ 
Typical Chiral HPLC for reactions with boronate $4 \mathbf{a}$.

Compound 21

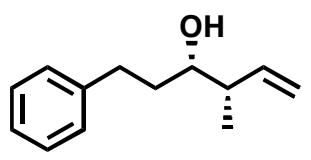

Racemic sample

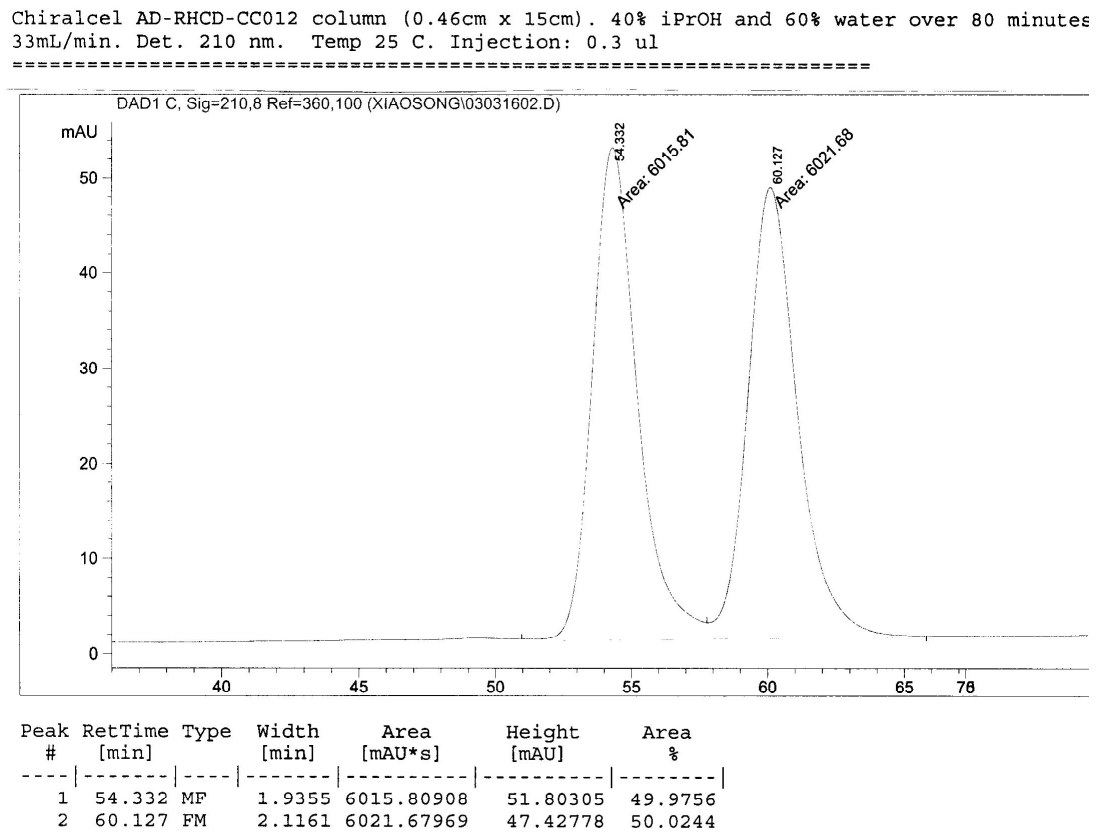

\section{Enantioselective reaction}

Chiralcel AD-RHCD-CC012 column $(0.46 \mathrm{~cm} \times 15 \mathrm{~cm})$. $40 \%$ iPrOH and $60 \%$ water over 80 minutes $33 \mathrm{~mL} / \mathrm{min}$. Det. $210 \mathrm{~nm}$. Temp $25 \mathrm{C}$. Injection: $0.3 \mathrm{ul}$

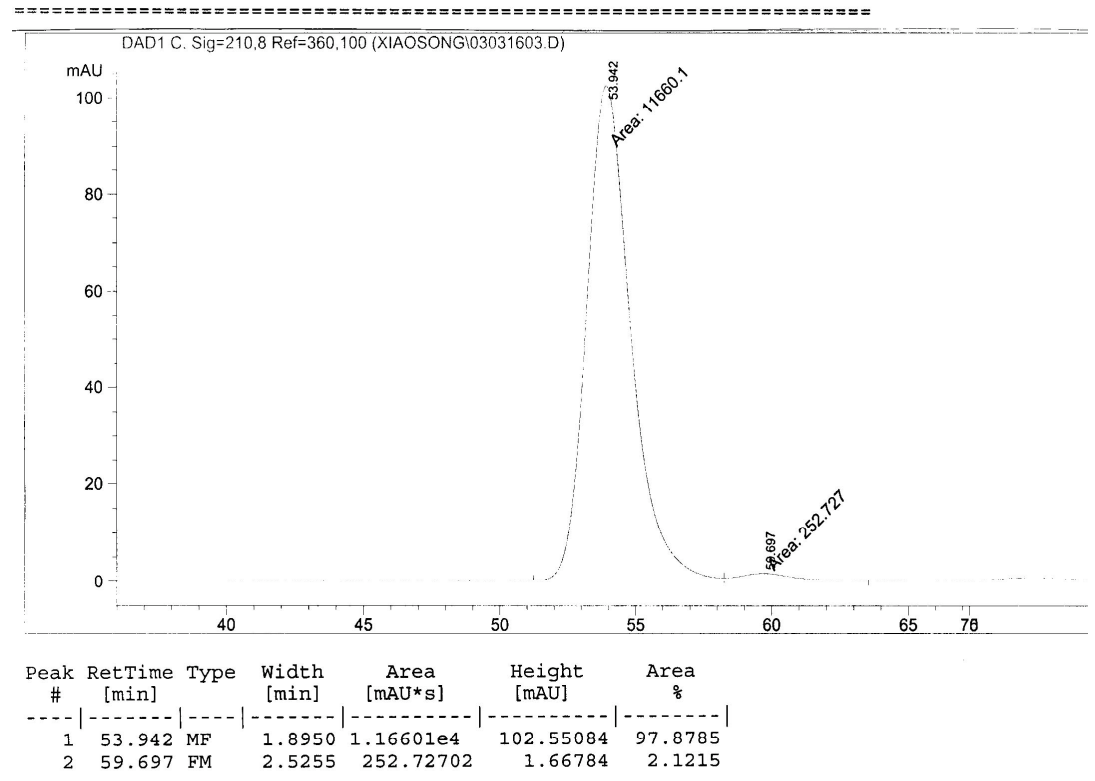




\section{Representative ${ }^{19} \mathbf{F}$ NMR spectra}

Typical ${ }^{19}$ F NMR for reactions with boronate $\mathbf{1 a}$

Compound 9
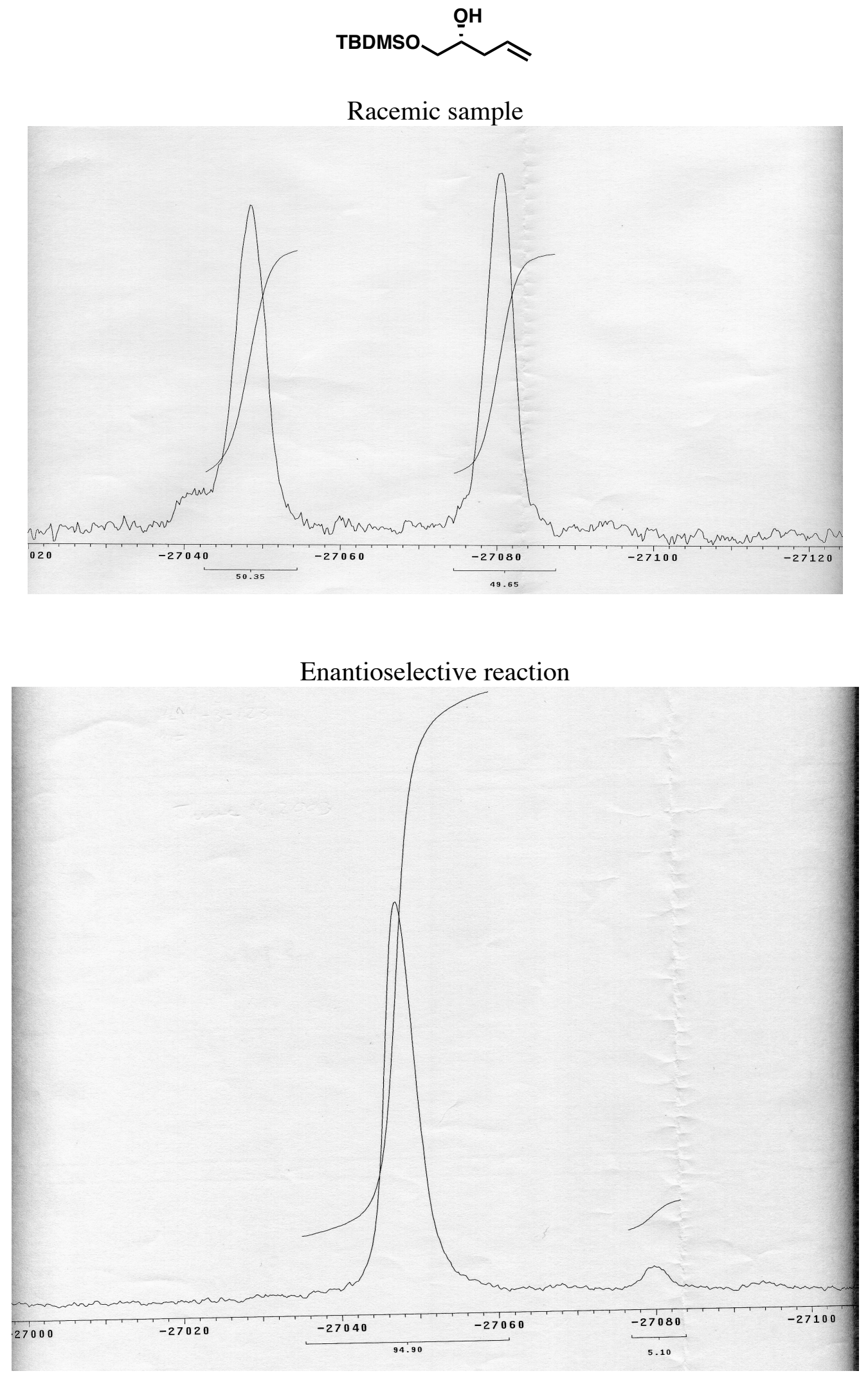